\title{
Computability of the causal boundary by using isocausality
}

\author{
J.L. Flores*, J. Herrera* ${ }^{* \dagger}$ M. Sánchez ${ }^{\dagger}$ \\ *Departamento de Álgebra, Geometría y Topología, \\ Facultad de Ciencias, Universidad de Málaga, \\ Campus Teatinos, 29071 Málaga, Spain \\ ${ }^{\dagger}$ Departamento de Geometría y Topología, \\ Facultad de Ciencias, Universidad de Granada, \\ Avenida Fuentenueva s/n, 18071 Granada, Spain
}

\begin{abstract}
Recently, a new viewpoint on the classical c-boundary in Mathematical Relativity has been developed, the relations of this boundary with the conformal one and other classical boundaries have been analyzed, and its computation in some classes of spacetimes, as the standard stationary ones, has been carried out.

In the present paper, we consider the notion of isocausality given by GarcíaParrado and Senovilla, and introduce a framework to carry out isocausal comparisons with standard stationary spacetimes. As a consequence, the qualitative behavior of the c-boundary (at the three levels: point set, chronology and topology) of a wide class of spacetimes, is obtained.
\end{abstract}

Keywords: causal boundary, causal map, isocausality, stationary spacetime, Cauchy completion, Busemann function, Finsler metric, Randers metric.

PACS: 04.20.Gz, 04.20.Ha

MSC2010: Primary 53C50, 83C75; Secondary 53C60, 53C80 


\section{Contents}

1 Introduction 3

2 Preliminaries 6

2.1 C-boundary of spacetimes . . . . . . . . . . . 8

2.2 Finsler Manifolds . . . . . . . . . . . . . . . . . . . . 11

2.3 Randers to stationary correspondence . . . . . . . . . . 12

3 Revisiting the c-boundary of stationary spacetimes 13

3.1 Relation between chronology and Busemann functions . . . . . . . 13

3.2 Point set structure of the partial boundaries . . . . . . . . . . . 15

3.3 Topology and S-relations . . . . . . . . . . . . . . . 16

3.4 Main result . . . . . . . . . . . . . . . . . 17

4 Framework for isocausal comparison 18

4.1 Causal mappings and c-boundary . . . . . . . . . . . . . 18

4.2 Spacetimes isocausal to standard stationary ones . . . . . . . . 19

5 Partial boundaries $\hat{\partial} V, \check{\partial} V$ through isocausality 20

5.1 Information on $\hat{\partial} V$ at the point set level . . . . . . . . . . . 21]

5.2 Discussion . . . . . . . . . . . . . . . . . . . 23

5.3 Strains and topological information . . . . . . . . . . . 25

5.4 Past c-boundary $\check{\partial} V \ldots \ldots \ldots \ldots \ldots \ldots$

6 The (total) c-boundary $\partial V$

$6.1 \partial V$ as a point set . . . . . . . . . . . . 33



6.3 Topology . . . . . . . . . . . . . . . . . . . 38

7 Conclusions 42 


\section{Introduction}

Recently, a new viewpoint and definition of the notion of causal boundary (cboundary, for short) $\partial V$ of a spacetime $V$ have been developed in [6]. In the case of spacetimes which are (conformal to) standard stationary ones, such a boundary has been extensively studied in [7, where the c-boundary is also carefully connected with other boundaries which appear naturally in Differential Geometry.

The machinery introduced in [6, 7] is enough to compute explicitly the c-boundary in many cases. Nevertheless, from a practical viewpoint, the c-boundary points may present quite a few of subtle (and bothering) possibilities for general spacetimes. This also happens in other simple boundaries. For example, the elementary Cauchy boundary of a (positive-definite) Riemannian manifold, regarded as a metric space, may be non-locally compact - and such a property can be transmitted to the cboundary. Of course, one does not expect that, in realistic spacetimes, all type of pathological mathematical properties will hold. In principle, a qualitative description of the c-boundary (with some criteria to understand at what extent some types of pathologies may exist) would be enough for many purposes. In the present article, this idea will be carried out by comparing the c-boundary of broad classes of spacetimes with the c-boundary of well-behaved standard stationary spacetimes. For this aim, the notion of isocausality in the sense introduced by García-Parrado and Senovilla [10. (see also [9, 11, 12, 13]) will be used sistematically.

Roughly speaking, two spacetimes are called isocausal when, by using a pair of diffeomorphisms, the timecones of the first one can be seen inside the timecones of the second one, and viceversa (see Definition 4.1). This includes the case of conformal equivalence (where a single diffeomorphism and its inverse are used) but isocausality is much more flexible. Even though this notion is simple, isocausality is also a subtle concept. For example, most of the steps of the so-called causal ladder of spacetimes are preserved by isocausal spacetimes [10 but not all of them [9. Section 3.2]. And, recently the authors exhibited an example of two isocausal spacetimes with different c-boundaries [5].

In spite of this last result (or perhaps because of it), we will show that the notion of isocausality is useful in order to analyze the c-boundary of some general classes of spacetimes. In fact, when a spacetime $V$ is isocausal to a simple standard stationary one $V_{c l}$ (whose c-boundary $\partial V_{c l}$ is known from [7]), a very interesting information on the c-boundary $\partial V$ of the original spacetime $V$ is obtained. Roughly speaking, $\partial V$ will be at least as rich as $\partial V_{c l}$. This means that $\partial V$ contains the elements of $\partial V_{c l}$ but perhaps some of these elements are "enlarged" - more precisely, $\partial V_{c l}$ is isomorphic to certain quotient of $\partial V$. Under simple hypotheses (expected to hold in the physically relevant cases) such enlargements are reasonably controlled.

This paper is organized as follows. In Section 2 we recall the notion of c-boundary introduced in [6], as well as some general elements of Finsler manifolds which are necessary for the computation of the c-boundary of a stationary spacetime. In Section 3 we consider the expression of a standard stationary spacetime endowed with an additional time dependent factor $\tilde{\alpha}(t)$. This expression may be interesting by itself (for example, in order to write the c-boundary when the condition 3.2 ) 
does not hold, extending the results in [7]). In this paper, such an expression will allow to make isocausal comparisons easily. For this purpose, we rewrite the known expressions of the c-boundary of a standard stationary spacetime (computed explicitly in $[7$ for the case $\tilde{\alpha}(t) \equiv 1)$ taking into account such a factor. In Section 4 , after explaining the notion of isocausality, the framework for isocausal comparison with a standard stationary spacetime is introduced. Choosing $\tilde{\alpha} \equiv 1$ and $\tilde{\alpha} \equiv \alpha<1$ we obtain two metrics $g_{c l}, g_{o p}$ which satisfy: (i) $g_{c l} \prec_{0} g_{o p}$, i.e., the timecones of the former are narrower than those of the latter, and (ii) both metrics are conformal and, thus, isocausal. Any metric $g$ with $g_{c l} \prec_{0} g \prec_{0} g_{o p}$ is then isocausal to $g_{c l}$ (and $g_{o p}$ ) and, so, our aim will be to relate the c-completions $\bar{V}_{c l}$ and $\bar{V}$ for $g_{c l}$ and $g$, resp. According to [7, as $g_{c l}$ is standard stationary, its c-boundary $\partial_{c l} V$ can be constructed by using a Finsler metric $F$ (the Fermat metric), which will be also compared in this section with a parameterized Finsler metric $F_{t}$ associated to $g$. In Section 5 the relation between the future boundaries $\hat{\partial}_{c l} V$ and $\hat{\partial} V$ (as well as between the past boundaries $\check{\partial}_{c l} V$ and $\check{\partial} V$ ) is analyzed, and in Section 6 the relation between the full c-boundaries $\partial_{c l} V$ and $\partial V$ is achieved. In the remainder of this Introduction, we give a heuristic explanation of these relations.

The possibility that two isocausal spacetimes may have different c-boundaries is explained carefully in [5. There, we considered three metrics $g_{c l} \prec_{0} g \prec_{0} g_{o p}$ on $\mathbb{R} \times \mathbb{R}^{-}$such that $g_{c l}$ and $g_{\text {op }}$ are conformal to the usual one $g_{0}=-d t^{2}+d x^{2}$. So, the c-boundary $\partial_{c l} V\left(\equiv \partial_{o p} V\right)$ is homeomorphic and naturally identifiable to the conformal boundary of $\left(\mathbb{R} \times \mathbb{R}^{-}, g_{0}\right)$ in $\mathbb{R}^{2}$, i.e. the line $x=0$, plus two lightlike lines that will be irrelevant for our discussion (Figure 1). The metric $g$ and its boundary $\partial V$ requires a somewhat involved construction (see the details in $[5]$ ). When one considers the boundary point $P_{0}=(0,0) \in \partial_{c l} V$, and try to relate it with some point of $\partial V$, one realizes that $P_{0}$ is stretched in a bigger piece of boundary, which we called the strain of $P_{0}, \operatorname{Str}\left(P_{0}\right)(\subset \partial V)$. In this particular example, $\operatorname{Str}\left(P_{0}\right)$ is naturally a lightlike segment (see Figure 2 left) but, in general, its structure may be more complicated. So, we prefer to represent it conceptually as in Figure 2 right. Obviously, one could refine this example, introducing more strains for other points, obtaining so the "skeleton" structure of Figure 3 . Clearly, $\partial V_{c l}$ is recovered by collapsing each strain of $\partial V$ to a single point.

In Sections 5, 6 we show at what extent this simple picture of $\partial V$ and $\partial_{c l} V$ holds in general. With this aim, we impose two type of hypothesis:

- Hypotheses to ensure that the metric $g$ is reasonably close to $g_{c l}$ and $g_{o p}$ for large $|t|$ : the integral conditions $(5.3)$ and $(5.15)$ on the factor $\alpha$ mentioned above (the former condition for the future boundary and the latter for the past one). These conditions are satisfied if, for example, $\alpha$ behaves as $1+$ $O\left(1 /|t|^{1+\epsilon}\right)$ for large $|t|$ and some $\epsilon>0$.

- Hypotheses to ensure that the model boundary $\partial_{c l} V$ is not too pathological. Concretely, the canonic generalized distance $d^{+}$of the Finsler metric associated to $g_{c l}$ will satisfy: (a) its Cauchy completion $M_{C}^{+}$is locally compact, and (b) the extension $d_{Q}^{+}$of $d^{+}$to the Cauchy boundary is still a generalized distance. For example, if we consider as the metric $g_{c l}$ a static one $(\omega \equiv 0$ 


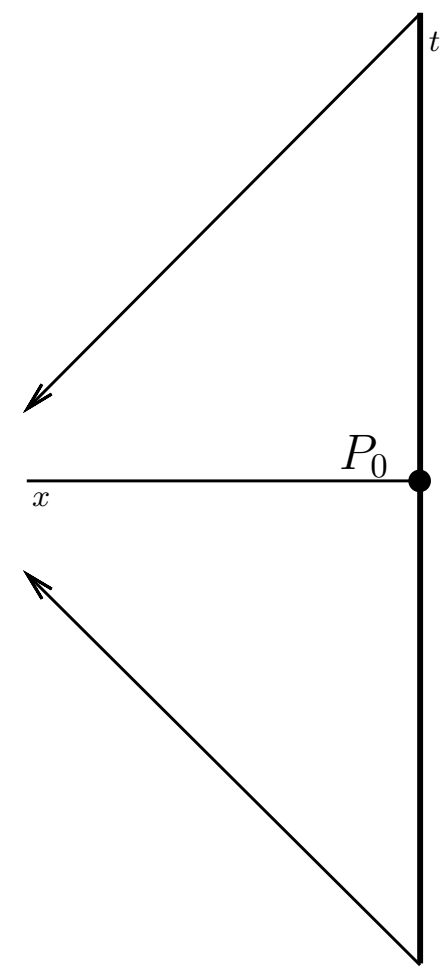

Figure 1: The c-boundary of $\left(\mathbb{R} \times \mathbb{R}^{-},-d t^{2}+d x^{2}\right)$.

The c-boundary is composed by a timelike line (the vertical segment) and two lightlike lines (which will not be relevant here). 


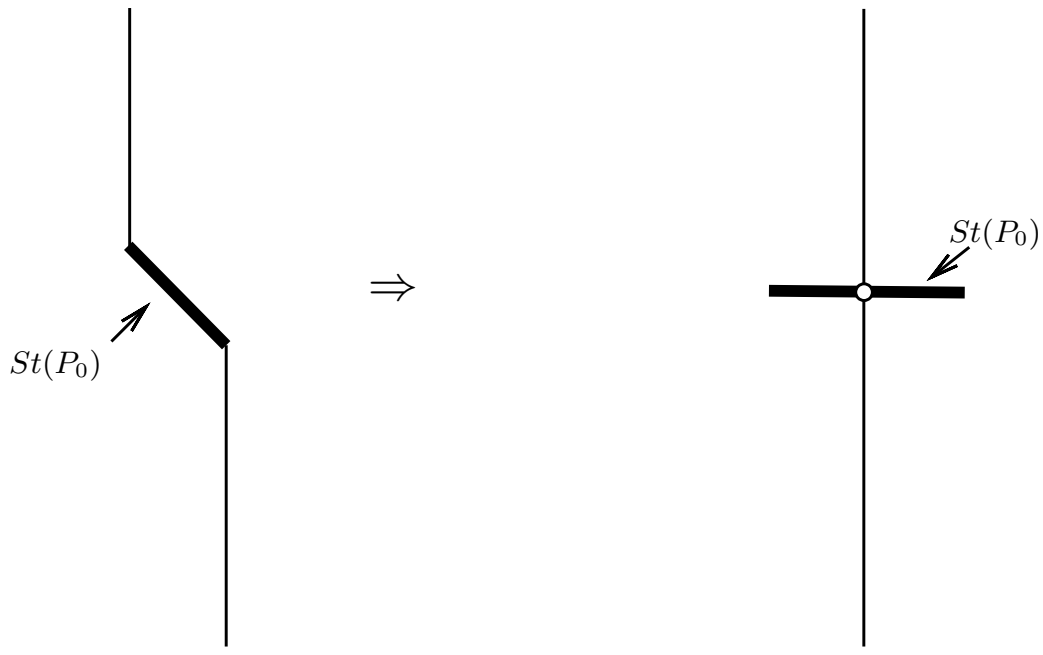

Figure 2: C-boundary of $(V, g)$.

The strain $\operatorname{St}\left(P_{0}\right)$ is drawn on the left as in [5] and on the right, more conceptually, as an horizontal segment (a "bone").

in formula (4.2), the Finsler metric becomes Riemannian, and the generalized distance $d^{+}$becomes a usual (symmetric) distance. So, condition (b) is automatically satisfied. On the other hand, condition (a) just prevents an undesirable property even for a Riemannian manifold (see the Riemannian example 4.9 and the Finslerian examples explained in Remark 3.26, both in reference [7, for some pathological samples where hypotheses (a) or (b) are not satisfied).

Under these hypotheses, in Section 5 we show that the partial boundaries $\partial \hat{\partial}, \partial \check{V}$ behave essentially as in the heuristic example above, that is, $\hat{\partial} V$ must contains $\hat{\partial}_{c l} V$ in the sense that a quotient $\hat{\partial} V / \sim_{s t}$ is identifiable to $\hat{\partial}_{c l} V$ (this occurs at two levels, as point set and topologically, recall Theorems 5.16, 5.18 and Remark 5.17). Finally, in Section 6 we show that these considerations can be extended to the (total) c-boundary (see Theorem 6.11. plus Remark 6.13 for some subtleties at the point set level). Moreover, this includes also considerations about the causal and chronological relations in the completions $\bar{V}, \bar{V}_{c l}$ which are related to the quotient space $\bar{V} / \sim$ (see Theorem 6.9 and Definition 6.10).

\section{Preliminaries}

Along this paper we will use typical background and terminology in Lorentzian Geometry as in [1, 15, 16. A spacetime $V$ will be a time-oriented connected smooth Lorentzian $(-,+, \ldots,+)$ manifold, also denoted $(V, g)$ abusing of notation. A tan- 




Figure 3: Conceptual view of the "skeleton" structure of the c-boundary.

The vertical segment ("column") represents the quotient space obtained by collapsing each strain (drawn as a horizontal one, a "bone"). This conceptual view of the strains as bones does not represent any topological nor causal structure as, in general, they could present different (and non-equivalent) structures (see Figure 5). 
gent vector $v \in T_{p} V, p \in V$ is called timelike (resp. lightlike; causal) if $g(v, v)<0$ (resp. $g(v, v)=0, v \neq 0 ; v$ is either timelike or lightlike). A causal vector is called future or past-directed if it belongs to the future or past cone. Accordingly, a smooth curve $\gamma: I \rightarrow V$ ( $I$ real interval) is called timelike, lightlike, causal and future or past-directed if so is $\dot{\gamma}(s)$ for all $s \in I$.

Two events $p, q \in V$ are chronologically related $p \ll q$ (resp. causally related $p \leqslant q$ ) if there exists some future-directed timelike (resp. either future-directed causal or constant) curve from $p$ to $q$. If $p \neq q$ and $p \leqslant q$ but $p \nless q$, then $p$ is said horismotically related to $q$. The chronological past (resp. future) of $p, I^{-}(p)$ (resp. $\left.I^{+}(p)\right)$ is defined as:

$$
I^{-}(p)=\{q \in V: q \ll p\} \quad\left(\text { resp. } I^{+}(p)=\{q \in V: p \ll q\}\right) .
$$

The chronological past $I^{-}(\gamma)=\cup_{s \in I} I^{-}(\gamma(s))$ (resp. future $I^{+}(\gamma)=\cup_{s \in I} I^{+}(\gamma(s))$ ) of future-directed (resp. past-directed) timelike curves $\gamma: I \rightarrow V$ will play a relevant role.

Along the paper several (time-oriented) Lorentzian metrics on the same manifold will be used. To avoid confusions, we will introduce a subindex to indicate the corresponding metric we are considering; say, $\ll_{\tilde{g}}$ or $I_{\tilde{g}}^{-}$for the metric $\tilde{g}$ on $V$.

\section{$2.1 \quad$ C-boundary of spacetimes}

The c-completion of spacetimes is constructed by adding ideal points to the spacetime in such a way that any timelike curve in the original spacetime acquires some endpoint in the new space 14. To formalize this construction, which will be conformally invariant and applicable to any strongly causal spacetime, previously we need to introduce some basic notions.

A non-empty subset $P \subset V$ is called a past set if it coincides with its past; i.e. $P=I^{-}(P):=\{p \in V: p \ll q$ for some $q \in P\}$. The common past of $S \subset V$ is defined by $\downarrow S:=I^{-}(\{p \in V: \quad p \ll q \forall q \in S\})$. In particular, the past and common past sets must be open. A past set that cannot be written as the union of two proper past sets is called indecomposable past set, IP. An IP which does coincide with the past of some point of the spacetime $P=I^{-}(p), p \in V$ is called proper indecomposable past set, PIP. Otherwise, $P=I^{-}(\gamma)$ for some inextendible future-directed timelike curve $\gamma$, and it is called terminal indecomposable past set, TIP. The dual notions of future set, common future, IF, TIF and PIF, are defined just by interchanging the roles of past and future in previous definitions.

To construct the future and past c-completion, first we have to identify each event $p \in V$ with its PIP, $I^{-}(p)$, and PIF, $I^{+}(p)$. This is possible in any distinguishing spacetime, that is, a spacetime which satisfies that two distinct events $p, q$ have distinct chronological futures and pasts $\left(p \neq q \Rightarrow I^{ \pm}(p) \neq I^{ \pm}(q)\right)$. In order to obtain consistent topologies in the c-completions, we will focus on a somewhat more restrictive class of spacetimes, the strongly causal ones. These are characterized by the fact that the PIPs and PIFs constitute a sub-basis for the topology of the manifold $V$. 
Now, every event $p \in V$ can be identified with its PIP, $I^{-}(p)$. So, the future c-boundary $\hat{\partial} V$ of $V$ is defined as the set of all the TIPs in $V$, and the future c-completion $\hat{V}$ becomes the set of all the IPs:

$$
V \equiv \mathrm{PIPs}, \quad \hat{\partial} V \equiv \mathrm{TIPs}, \quad \hat{V} \equiv \operatorname{IPs} .
$$

Analogously, each $p \in V$ can be identified with its $\mathrm{PIF}, I^{+}(p)$. The past c-boundary $\check{\partial} V$ of $V$ is defined as the set of all the TIFs in $V$, and the past c-completion $\check{V}$ is the set of all the IFs:

$$
V \equiv \mathrm{PIFs}, \quad \check{\partial} V \equiv \mathrm{TIFs}, \quad \check{V} \equiv \mathrm{IFs} .
$$

For the (total) c-boundary, the so-called S-relation comes into play 17. Denote $\hat{V}_{\emptyset}=\hat{V} \cup\{\emptyset\}$ (resp. $\left.\check{V}_{\emptyset}=\breve{V} \cup\{\emptyset\}\right)$. The S-relation $\sim_{S}$ is defined in $\hat{V}_{\emptyset} \times \check{V}_{\emptyset}$ as follows. First, in the case $(P, F) \in \hat{V} \times \check{V}$,

$$
P \sim_{S} F \Longleftrightarrow\left\{\begin{array}{lll}
P & \text { is included and is a maximal IP into } & \downarrow F \\
F & \text { is included and is a maximal IF into } & \uparrow P .
\end{array}\right.
$$

By maximal we mean that no other $P^{\prime} \in \hat{V}$ (resp. $F^{\prime} \in \check{V}$ ) satisfies the stated property and includes strictly $P$ (resp. $F$ ). Recall that, as proved by Szabados [17, $I^{-}(p) \sim_{S} I^{+}(p)$ for all $p \in V$, and these are the unique S-relations (according to our definition (2.1) involving proper indecomposable sets. Now, in the case $(P, F) \in \hat{V}_{\emptyset} \times \check{V}_{\emptyset} \backslash\{(\emptyset, \emptyset)\}$, we also put

$$
P \sim_{S} \emptyset, \quad\left(\operatorname{resp} . \emptyset \sim_{S} F\right)
$$

if $P$ (resp. $F$ ) is a (non-empty, necessarily terminal) indecomposable past (resp. future) set that is not S-related by (2.1) to any other indecomposable set; notice that $\emptyset$ is never S-related to itself. Now, we can introduce the notion of c-completion, according to [6]:

Definition 2.1 As a point set, the c-completion $\bar{V}$ of a strongly causal spacetime $V$ is formed by all the pairs $(P, F) \in \hat{V}_{\emptyset} \times \check{V}_{\emptyset}$ such that $P \sim_{S} F$. The c-boundary $\partial V$ is defined as $\partial V:=\bar{V} \backslash V$, where $V \equiv\left\{\left(I^{-}(p), I^{+}(p)\right): p \in V\right\}$.

The chronological relation $\ll$ of the spacetime is extended to the c-completion in the following way. We say that $(P, F),\left(P^{\prime}, F^{\prime}\right) \in \bar{V}$ are chronologically related, $(P, F) \ll\left(P^{\prime}, F^{\prime}\right)$, if $F \cap P^{\prime} \neq \emptyset$. Regarding to the extension of the causal relation $\leqslant$, it is enough to consider the following sufficient criterium for $(P, F),\left(P^{\prime}, F^{\prime}\right) \in \bar{V}$ with, either $P \neq \emptyset$ or $F^{\prime} \neq \emptyset$ :

$$
P \subset P^{\prime} \text { and } F^{\prime} \subset F \Rightarrow(P, F) \leqslant\left(P^{\prime}, F^{\prime}\right) .
$$

In the particular case of the spacetimes treated in this paper (see Section 4.2), this criterium can be also regarded as the general definition of $\leqslant$ in $\bar{V}$ (recall the discussion in [7, Sect. 6.4] and references therein). Moreover, we will say that two 
different pairs in $\bar{V}$ are horismotically related if they are causally but not chronologically related.

Finally, the topology of the spacetime is also extended to the c-completion by means of the so-called chronological topology (chr. topology, for short). In order to define it, first consider the following limit operator $L$ for $\bar{V}$ : given a sequence $\sigma=\left\{\left(P_{n}, F_{n}\right)\right\} \subset \bar{V}$,

$$
(P, F) \in L(\sigma) \Longleftrightarrow\left\{\begin{array}{lll}
P \in \hat{L}\left(\left\{P_{n}\right\}\right) & \text { whenever } \quad P \neq \emptyset \\
F \in \check{L}\left(\left\{F_{n}\right\}\right) & \text { whenever } \quad F \neq \emptyset
\end{array}\right.
$$

where

$\hat{L}\left(\left\{P_{n}\right\}\right):=\left\{P^{\prime} \in \hat{V}: P^{\prime} \subset \operatorname{LI}\left(\left\{P_{n}\right\}\right)\right.$ and $P^{\prime}$ is a maximal IP into $\left.\operatorname{LS}\left(\left\{P_{n}\right\}\right)\right\}$

$\check{L}\left(\left\{F_{n}\right\}\right):=\left\{F^{\prime} \in \check{V}: F^{\prime} \subset \operatorname{LI}\left(\left\{F_{n}\right\}\right)\right.$ and $F^{\prime}$ is a maximal IF into $\left.\operatorname{LS}\left(\left\{F_{n}\right\}\right)\right\}$

(LI and LS are the usual point set inferior and superior limits of sets). Then, one can check that a topology is defined on $\bar{V}$ as follows:

$C$ is closed $\Leftrightarrow L(\sigma) \subset C$ for any sequence $\sigma \subset C$.

Note that a topology on the future (resp. past) c-completion $\hat{V}$ (resp. $\check{V}$ ) can be defined in a similar way, just by using the limit operator $\hat{L}$ (resp. $\check{L}$ ) instead of $L$. In this case, the resulting topology, which also extends the topology of the spacetime, is called the future (resp. past) chronological topology.

Remark 2.2 We remark the following basic properties about the chronological topology:

(1) The chronological topology (as well as the future and past ones) is sequential and $T_{1}$ (see [6, Prop. 3.39 and 3.21]), but may be non-Hausdorff.

(2) Clearly, if $(P, F) \in L\left(\left\{\left(P_{n}, F_{n}\right)\right\}\right)$ then $\left\{\left(P_{n}, F_{n}\right)\right\}$ converges to $(P, F)$. When the converse happens, $L$ is called of first order (see [6, Section 3.6]).

(3) Given a pair $(P, F) \in \partial V$, any timelike curve defining $P$ (or $F$ ) converges to $(P, F)$ with the chronological topology (see [6, Th. 3.27]).

These definitions for the c-boundary construction involve some particular subtleties, which are essentially associated to the following two facts: first, a TIP (or TIF) may not determine a unique pair in the c-boundary, and, second, the topology does not always agree with the S-relation, in the sense that, for S-related elements as above

$$
P \in \hat{L}\left(P_{n}\right) \nLeftarrow F \in \check{L}\left(F_{n}\right) .
$$

This makes natural to remark the following special cases:

Definition 2.3 A spacetime $V$ has a c-completion $\bar{V}$ which is simple as a point set if each TIP (resp. each TIF) determines a unique pair in $\partial V$.

Moreover, the c-completion is simple if it is simple as a point set and also topologically simple, i.e. $(P, F) \in L\left(P_{n}, F_{n}\right)$ holds when either $P \in \hat{L}\left(\left\{P_{n}\right\}\right)$ or $F \in \check{L}\left(\left\{F_{n}\right\}\right)$. 
Remark 2.4 The previous definition is slightly redundant. Even though the notion of simple comprises two levels, simplicity as a point set and as a topological set, really it is equivalent to the second level. In fact, if the c-completion is topologically simple and we assume by contradiction the existence of, say, $\left(P, F_{1}\right),\left(P, F_{2}\right) \in$ $\partial V$ with $F_{1} \neq F_{2}$, then the constant sequence $\left\{\left(P, F_{1}\right)\right\}$ converges to $\left(P, F_{1}\right)$ and not to $\left(P, F_{2}\right)$ (as the c-completion is always $\left.T_{1}\right)$ in contradiction with topological simplicity.

\section{$2.2 \quad$ Finsler Manifolds}

A Finsler metric $F$ on a manifold $M$ gives smoothly a positively homogeneous norm at each $p \in M$, where positive homogeneity means that the usual equality $F(\lambda v)=$ $|\lambda| F(v)$ for $v \in T M$ of the usual norms is assumed only when $\lambda \geq 0$. So, given such a $F$, one can define the reverse Finsler metric: $F^{\mathrm{rev}}(v):=F(-v)$. Given a Finsler manifold $(M, F)$, a map $d: M \times M \rightarrow \mathbb{R}$ is defined in the following way:

$$
d(x, y):=\inf _{c \in C(x, y)} \operatorname{length}(c)=\inf _{c \in C(x, y)} \int_{s_{0}}^{s_{1}} F(\dot{c}(s)) d s,
$$

where $C(x, y)$ is the set of piecewise smooth curves $c:\left[s_{0}, s_{1}\right] \rightarrow M$ with $c\left(s_{0}\right)=x$, $c\left(s_{1}\right)=y$. Such a $d$ is a generalized distance, that is, it satisfies all the axioms of a distance except symmetry (i.e. $d$ is a quasi-distance) and, additionally, the following condition holds: a sequence $\left\{x_{n}\right\} \subset M$ satisfies $d\left(x, x_{n}\right) \rightarrow 0$ iff $d\left(x_{n}, x\right) \rightarrow 0$. One can define the forward and backward open balls of center $x_{0} \in M$ and radius $r>0$ as: $B^{+}\left(x_{0}, r\right)=\left\{x \in M: d\left(x_{0}, x\right)<r\right\}$ and $B^{-}\left(x_{0}, r\right)=\left\{x \in M: d\left(x, x_{0}\right)<r\right\}$, resp. Each type of balls constitutes a topological basis of $M$.

A sequence $\sigma=\left\{x_{n}\right\}$ in $(M, F)$ is a (forward) Cauchy sequence if for all $\epsilon>0$ there exists $n_{0}$ such that $d\left(x_{n}, x_{m}\right)<\epsilon$ whenever $n_{0} \leqslant n \leqslant m$. Let $\operatorname{Cau}(M)$ be the space of all the Cauchy sequences in $(M, F)$. Two Cauchy sequences $\sigma, \sigma^{\prime} \in$ $\operatorname{Cau}(M)$, with $\sigma=\left\{x_{n}\right\}, \sigma^{\prime}=\left\{x_{n}^{\prime}\right\}$, are related $\sigma \sim \sigma^{\prime}$ iff:

$$
\lim _{n} \lim _{m} d\left(x_{n}, x_{m}^{\prime}\right)=\lim _{n} \lim _{m} d\left(x_{n}^{\prime}, x_{m}\right)=0 .
$$

With these notions at hand, the (forward) Cauchy completion $M_{C}^{+}$and (forward) Cauchy boundary $\partial_{C}^{+} M$ of $(M, F)$ are defined by:

$$
M_{C}^{+}=\mathrm{Cau}(M) / \sim \quad \partial_{C}^{+} M:=M_{C}^{+} \backslash M .
$$

The backward Cauchy sequence, its correspondence space $\mathrm{Cau}^{\mathrm{rev}}(M)$, and consequently, the backward Cauchy completion $M_{C}^{-}$and the backward Cauchy boundary $\partial_{C}^{-} M$, are defined analogously but using $d^{\text {rev }}$, defined as $d^{\text {rev }}(x, y):=d(y, x)$ for all $x, y$. Note that $d^{\text {rev }}$ can be also obtained from 2.4 by replacing $F$ by $F^{\text {rev }}$.

The generalized distance $d$ (resp. $d^{\text {rev }}$ ) on $M$ can be extended to a quasi-distance $d_{Q}: M_{C}^{+} \times M_{C}^{+} \rightarrow[0, \infty]\left(\right.$ resp. $\left.d_{Q}^{\mathrm{rev}}: M_{C}^{-} \times M_{C}^{-} \rightarrow[0, \infty]\right)$ in a natural way, i.e.

$$
d_{Q}\left(\left[\left\{x_{n}\right\}\right],\left[\left\{y_{n}\right\}\right]\right):=\lim _{n}\left(\lim _{m} d\left(x_{n}, y_{m}\right)\right)
$$

$$
\left(\text { resp. } d_{Q}^{\text {rev }}\left(\left[\left\{x_{n}\right\}\right],\left[\left\{y_{n}\right\}\right]\right):=\lim _{n}\left(\lim _{m} d^{\text {rev }}\left(x_{n}, y_{m}\right)\right)\right) \text {. }
$$


In general, as $d_{Q}$ (resp. $\left.d_{Q}^{\text {rev }}\right)$ is not a generalized distance (see [7, Example 3.24]), it generates two (in general, different) topologies on $M_{C}^{+}$(resp. $M_{C}^{-}$): one by using the corresponding forward balls and the other one by using the backward ones. The natural one, so that forward (resp. backward) Cauchy sequences converge to the point represented by its class in $M_{C}^{+}$(resp. $M_{C}^{-}$), is the generated by the backward (resp. forward) balls.

With this choice the natural inclusion $i: M \hookrightarrow M_{C}^{+}$(resp. $i: M \hookrightarrow M_{C}^{-}$) becomes a topological embedding, being $\partial_{C}^{+} M$ (resp. $\left.\partial_{C}^{-} M\right)$ a closed subset of $M_{C}^{+}$ (resp. $M_{C}^{-}$). The topology associated to $d_{Q}$ (resp. $d_{Q}^{\text {rev }}$ ) is not Hausdorff (neither $\left.T_{1}\right)$ in general, but only $T_{0}$. On the other hand, the distance $d_{Q}$ (resp. $d_{Q}^{\text {rev }}$ ) can be extended (with the same formal definition) to $d_{Q}: M_{C}^{+} \times\left(M_{C}^{+} \cup M_{C}^{-}\right) \rightarrow[0, \infty]$ (resp. $\left.d_{Q}^{\text {rev }}:\left(M_{C}^{+} \cup M_{C}^{-}\right) \times M_{C}^{-} \rightarrow[0, \infty]\right)$.

The Cauchy boundaries $\partial_{C}^{+} M, \partial_{C}^{-} M$ can be related thanks to the following property: if $\sigma, \sigma^{\prime} \in \mathrm{Cau}(M)$ are related for $d$, and $\sigma \in \mathrm{Cau}^{\mathrm{rev}}(M)$, then $\sigma^{\prime} \in \mathrm{Cau}^{\mathrm{rev}}(M)$ and $\sigma, \sigma^{\prime}$ are related for $d^{\text {rev }}$ ([7, Prop. 3.20]). So, one can define the symmetrized Cauchy boundary as the following intersection:

$$
\partial_{C}^{s} M=\partial_{C}^{+} M \cap \partial_{C}^{-} M .
$$

Moreover, a sequence $\sigma \in \partial_{C}^{s} M$ iff it is a Cauchy sequence (in the classical sense) for the symmetrized distance

$$
d^{s}(x, y):=\frac{d(x, y)+d^{\mathrm{rev}}(x, y)}{2},
$$

which is a (true) distance, even though it cannot be obtained as a length space in general (i.e. as the infimum of lengths of connecting curves). On $M_{C}^{s}=M \cup \partial_{C}^{s} M$ one can also define the extension $\bar{d}^{s}$ of $d^{s}$ on $M$, which satisfies $\bar{d}^{s}=\left(d_{Q}+d_{Q}^{\text {rev }}\right) / 2$. All the relevant properties of these constructions are summarized in [7, Theor. 1.1].

\subsection{Randers to stationary correspondence}

Taking into account the conformal invariance of the c-boundary, we consider, without loss of generality, the normalized expression (2.6) below as the definition of any standard stationary spacetime:

$$
V=\left(\mathbb{R} \times M, g=-d t^{2}+\pi_{M}^{*} \omega \otimes d t+d t \otimes \pi_{M}^{*} \omega+\pi_{M}^{*} h\right),
$$

where $\omega$ is a 1 -form, $h$ is a Riemannian metric, both on $M$, and $\pi_{M}: \mathbb{R} \times M \rightarrow M$, $t: \mathbb{R} \times M \rightarrow \mathbb{R}$ are the natural projections. The elements in (2.6) allow to construct the following Finsler metrics (of Randers type) on $M$ :

$$
\begin{aligned}
& F^{+}(v)(=F(v))=\sqrt{h(v, v)+\omega(v)^{2}}+\omega(v), \\
& F^{-}(v)\left(=F^{\mathrm{rev}}(v)\right)=\sqrt{h(v, v)+\omega(v)^{2}}-\omega(v) .
\end{aligned}
$$

We will denote by length ${ }_{ \pm}$and $d^{ \pm}$, resp., the generalized length and distance associated to $F^{ \pm}$(as in 2.4$)$, and by $d_{Q}^{ \pm}$the natural extension of $d^{ \pm}$to $M_{C}^{ \pm}$. 
In 2 the authors analyzed the relation between different properties of stationary spacetimes and these Finsler metrics of Randers type. In [7] this study was extended to the c-boundary. In the next section we revisit this second study by reproducing it in a slightly more general framework.

\section{Revisiting the c-boundary of stationary space- times}

Consider a connected Riemannian manifold $(M, h)$, a 1-form $\omega$ and the natural projections $\pi_{M}, t$ as above. For each smooth positive function $\tilde{\alpha}: \mathbb{R} \rightarrow \mathbb{R}$, consider the spacetime $V_{\tilde{\alpha}}:=\left(\mathbb{R} \times M, g_{\tilde{\alpha}}\right)$, with

$$
g_{\tilde{\alpha}}:=-d t^{2}+\tilde{\alpha}(t) \pi_{M}^{*} \omega \otimes d t+\tilde{\alpha}(t) d t \otimes \pi_{M}^{*} \omega+\tilde{\alpha}^{2}(t) \pi_{M}^{*} h,
$$

where the pull-back $\pi_{M}^{*}$ will be omitted when there is no possibility of confusion and $\tilde{\alpha}(t)$ denotes $\tilde{\alpha} \circ t$ (the future time-orientation is always determined by $\partial_{t}$ ). Up to the conformal factor $\tilde{\alpha}^{2}(t)$, the change of variables $d s=d t / \tilde{\alpha}(t)$ allows to identify the spacetime $V_{\tilde{\alpha}}$ with a region $I \times M(I \subset \mathbb{R}$ interval, $0 \in I)$ of the standard stationary spacetime (2.6). Moreover, this region $I \times M$ becomes the full standard stationary spacetime (i.e., $I=\mathbb{R}$ ) if and only if the following condition holds (see the proof of Proposition 4.6 for details):

$$
\int_{t=0}^{t=\infty} \frac{d s}{\tilde{\alpha}(s)}=\int_{t=-\infty}^{t=0} \frac{d s}{\tilde{\alpha}(s)}=\infty
$$

The results in [7, Sect. 6] determine accurately the c-boundary of any standard stationary spacetime, and thus, under condition 3.2 , the c-boundary of the spacetime $V_{\tilde{\alpha}}$ as well. Next, we will translate the results in this reference to describe the c-boundary of $V_{\tilde{\alpha}}$ maintaining explicitly the dependence on $\tilde{\alpha}$ (which will be useful for the isocausal comparison). We will assume (3.2) throughout all the paper, even though the steps in Subsection 3.1 do not require it.

\subsection{Relation between chronology and Busemann functions}

Let us begin by establishing the following characterization of the chronological relation in $V_{\tilde{\alpha}}$ (see [7, Prop. 6.1]):

Proposition 3.1 If $\gamma(t)=(t, c(t)), t \in[a, \Omega)$, is a future-directed timelike curve in $V_{\tilde{\alpha}}=\left(\mathbb{R} \times M, g_{\tilde{\alpha}}\right)$. Then $F^{+}(\dot{c}(t))<1 / \tilde{\alpha}(t)$ and length ${ }_{+}\left(\left.c\right|_{\left[t_{0}, t_{1}\right]}\right)<\int_{t_{0}}^{t_{1}} d s / \tilde{\alpha}(s)$, with $\alpha \leqslant t_{0} \leqslant t_{1}<\Omega$. Moreover,

$$
\left(t_{0}, x_{0}\right) \ll_{\tilde{\alpha}}\left(t_{1}, x_{1}\right) \Longleftrightarrow d^{+}\left(x_{0}, x_{1}\right)<\int_{t_{0}}^{t_{1}} \frac{d s}{\tilde{\alpha}(s)} .
$$

As a direct consequence, note that $\int_{t_{0}}^{t} d s / \tilde{\alpha}(s)-d(\cdot, c(t))$ increases with $t$. 
According to Section 2.1, any IP in $\left(\mathbb{R} \times M, g_{\tilde{\alpha}}\right)$ is of the form $P_{\tilde{\alpha}}=I_{\tilde{\alpha}}^{-}(\gamma)$, being $\gamma$ a future-directed timelike curve with $\gamma(t)=(t, c(t))$ and $t \in[a, \Omega)$. Then, from Proposition 3.1 one deduces

$$
\begin{aligned}
P_{\tilde{\alpha}} & =I_{\tilde{\alpha}}^{-}(\gamma) \\
& =\left\{\left(t_{0}, x_{0}\right) \in V_{\tilde{\alpha}}:\left(t_{0}, x_{0}\right) \ll_{\tilde{\alpha}}(t, c(t)) \text { for some } t \text { close enough to } \Omega\right\} \\
& =\left\{\left(t_{0}, x_{0}\right) \in V_{\tilde{\alpha}}: 0<\lim _{t \rightarrow \Omega}\left(\int_{t_{0}}^{t} \frac{d s}{\tilde{\alpha}(s)}-d^{+}\left(x_{0}, c(t)\right)\right)\right\} \\
& =\left\{\left(t_{0}, x_{0}\right) \in V_{\tilde{\alpha}}: \int_{0}^{t_{0}} \frac{d s}{\tilde{\alpha}(s)}<\lim _{t \rightarrow \Omega}\left(\int_{0}^{t} \frac{d s}{\tilde{\alpha}(s)}-d^{+}\left(x_{0}, c(t)\right)\right)\right\} .
\end{aligned}
$$

Therefore, if one defines the forward Busemann function for $g_{\tilde{\alpha}}$ associated to a curve $c$ with $F^{+}(\dot{c}(t))<1 / \tilde{\alpha}(t)$ as

$$
b_{c, \tilde{\alpha}}^{+}(\cdot):=\lim _{t \rightarrow \Omega}\left(\int_{0}^{t} \frac{d s}{\tilde{\alpha}(s)}-d^{+}(\cdot, c(t))\right),
$$

one can write $P_{\tilde{\alpha}}=P_{\tilde{\alpha}}\left(b_{c, \tilde{\alpha}}^{+}\right)$where, for any function $f$,

$$
P_{\tilde{\alpha}}(f)=\left\{\left(t_{0}, x_{0}\right) \in V_{\tilde{\alpha}}: \int_{0}^{t_{0}} \frac{d s}{\tilde{\alpha}(s)}<f\left(x_{0}\right)\right\} .
$$

Similarly, any IF in $V_{\tilde{\alpha}}$ is of the form $F_{\tilde{\alpha}}=I_{\tilde{\alpha}}^{+}(\gamma)$, being $\gamma$ a past-directed timelike curve with $\gamma(t)=(-t, c(t))$ and $t \in[a,-\Omega)$. Thus, $F_{\tilde{\alpha}}=F_{\tilde{\alpha}}\left(b_{c, \tilde{\alpha}}^{-}\right)$where

$$
F_{\tilde{\alpha}}(f)=\left\{\left(t_{0}, x_{0}\right) \in V_{\tilde{\alpha}}: \int_{0}^{t_{0}} \frac{d s}{\tilde{\alpha}(s)}>f\left(x_{0}\right)\right\},
$$

and

$$
b_{c, \tilde{\alpha}}^{-}(\cdot):=\lim _{t \rightarrow-\Omega}\left(-\int_{-t}^{0} \frac{d s}{\tilde{\alpha}(s)}+d^{-}(\cdot, c(t))\right)=\lim _{t \rightarrow-\Omega}\left(\int_{0}^{-t} \frac{d s}{\tilde{\alpha}(s)}+d^{-}(\cdot, c(t))\right)
$$

is the backward Busemann function for $g_{\tilde{\alpha}}$ associated to a curve $c$ with $F^{-}(\dot{c}(t))<$ $1 / \tilde{\alpha}(t)$. In conclusion, the set of all IPs (resp. IFs) can be identified with the set of all forward (resp. backward) Busemann functions. So, denoting as $B_{\tilde{\alpha}}^{+}(M)$ (resp. $\left.B_{\tilde{\alpha}}^{-}(M)\right)$ the set of all the finite forward (resp. backward) Busemann functions, one has 1

$$
\hat{V}_{\tilde{\alpha}} \equiv B_{\tilde{\alpha}}^{+}(M) \cup\{\infty\} \quad \check{V}_{\tilde{\alpha}} \equiv B_{\tilde{\alpha}}^{-}(M) \cup\{-\infty\} .
$$

Next, assume $\pm \Omega<\infty$. From Prop. 3.1. length $_{ \pm}\left(\left.c\right|_{[a, \pm \Omega)}\right)<\int_{a}^{ \pm \Omega} d s / \tilde{\alpha}(s)<\infty$, and so, $c(t) \rightarrow x^{ \pm}$for some $x^{ \pm} \in M_{C}^{ \pm}$. In particular, for each $\Omega \in \mathbb{R}$, putting $\Omega_{\tilde{\alpha}}:=\int_{0}^{\Omega} d s / \tilde{\alpha}(s)$ :

$$
b_{c, \tilde{\alpha}}^{ \pm}(\cdot)=\int_{0}^{\Omega} \frac{d s}{\tilde{\alpha}(s)} \mp d_{Q}^{ \pm}\left(\cdot, x^{ \pm}\right)=\Omega_{\tilde{\alpha}} \mp d_{Q}^{ \pm}\left(\cdot, x^{ \pm}\right), \quad x^{ \pm} \in M_{C}^{ \pm} .
$$

\footnotetext{
${ }^{1}$ Note that a forward (resp. backward) Busemann function takes the value plus (resp. minus) infinity at some point iff it is constantly equal to plus (resp. minus) infinity.
} 
Therefore, if we define

$$
d_{\left(\Omega_{\tilde{\alpha}}, x^{ \pm}\right)}^{ \pm}(\cdot):=\Omega_{\tilde{\alpha}} \mp d_{Q}^{ \pm}\left(\cdot, x^{ \pm}\right) \quad \text { for any } x^{ \pm} \in M_{C}^{ \pm},
$$

we have just deduced that

$$
b_{c, \tilde{\alpha}}^{ \pm}(\cdot)=d_{\left(\Omega_{\tilde{\alpha}, x^{ \pm}}^{ \pm}\right.}^{ \pm}(\cdot) \quad \text { if } \quad \pm \Omega<\infty .
$$

\subsection{Point set structure of the partial boundaries}

In the remainder of this section, we will describe the c-boundary of $V_{\tilde{\alpha}}$ for $I=\mathbb{R}$, i.e., under condition 3.2 . So, the following action on $B_{\tilde{\alpha}}^{ \pm}(M)$ is well-defined:

$$
\begin{aligned}
& B_{\tilde{\alpha}}^{ \pm}(M) \times \mathbb{R} \quad \rightarrow \quad B_{\tilde{\alpha}}^{ \pm}(M) \\
& \left(b_{c, \tilde{\alpha}}^{ \pm}, K\right) \mapsto b_{c, \tilde{\alpha}}^{ \pm}+K .
\end{aligned}
$$

In fact, (for the case ${ }^{+}$) standard arguments allow one to deduce from condition (3.2) the existence of some increasing bijective smooth function $s_{K}(t)$ such that:

$$
\int_{t}^{s_{K}(t)} \frac{1}{\tilde{\alpha}(r)} d r=K, \quad \text { and so } \quad \dot{s}_{K}(t)=\frac{\tilde{\alpha}\left(s_{K}(t)\right)}{\tilde{\alpha}(t)} .
$$

As a consequence,

$$
\begin{aligned}
b_{c, \tilde{\alpha}}^{+}+K & =\lim _{t \rightarrow \infty}\left(\int_{0}^{t} \frac{d r}{\tilde{\alpha}(r)}-d^{+}(\cdot, c(t))\right)+K \\
& =\lim _{t \rightarrow \infty}\left(\int_{0}^{t} \frac{d r}{\tilde{\alpha}(r)}+\int_{t}^{s_{K}(t)} \frac{d r}{\tilde{\alpha}(r)}-d^{+}(\cdot, c(t))\right) \\
& =\lim _{t \rightarrow \infty}\left(\int_{0}^{s_{K}(t)} \frac{d r}{\tilde{\tilde{\alpha}}(r)}-d^{+}(\cdot, c(t))\right) \\
& =\lim _{s \rightarrow \infty}\left(\int_{0}^{s} \frac{d r}{\tilde{\alpha}(r)}-d^{+}(\cdot, \bar{c}(s))\right), \quad \text { where } \bar{c}\left(s_{K}(t)\right):=c(t),
\end{aligned}
$$

and notice that

$$
F\left(\dot{\bar{c}}\left(s_{K}(t)\right)\right)=F\left(\dot{c}(t) / \dot{s}_{K}(t)\right)=\frac{F(\dot{c}(t))}{\dot{s}_{K}(t)}<\frac{1}{\tilde{\alpha}(t)} \cdot \frac{\tilde{\alpha}(t)}{\tilde{\alpha}\left(s_{K}(t)\right)}=\frac{1}{\tilde{\alpha}\left(s_{K}(t)\right)} .
$$

So, the last term in 3.11) (under inequality 3.12 for $\bar{c}$ ) is just the definition of $b_{\bar{c}, \tilde{\alpha}}^{+} \in B_{\tilde{\alpha}}^{+}(M)$, as required.

Next, define the forward/backward Busemann completion as the quotient space $M_{B, \tilde{\alpha}}^{ \pm}=B_{\tilde{\alpha}}^{ \pm}(M) / \mathbb{R}$ provided by the action 3.10 . From 3.9 , the forward/backward Busemann completion $M_{B, \tilde{\alpha}}^{ \pm}$clearly contains the forward/backward Cauchy completion $M_{C}^{ \pm}$. So, if we define the forward/backward Busemann boundary as $\partial_{B, \tilde{\alpha}}^{ \pm} M:=$ $M_{B, \tilde{\alpha}}^{ \pm} \backslash M$ and the forward/backward proper Busemann boundary as $\partial_{\mathcal{B}, \tilde{\alpha}}^{ \pm} M:=$ $\partial_{B, \tilde{\alpha}}^{ \pm} M \backslash \partial_{C}^{ \pm} M$, one obtains the decomposition:

$$
M_{B, \tilde{\alpha}}^{ \pm}=M \cup \partial_{B, \tilde{\alpha}}^{ \pm} M=M \cup \partial_{C}^{ \pm} M \cup \partial_{\mathcal{B}, \tilde{\alpha}}^{ \pm} M
$$


Finally, labelling the TIP (resp. TIF) $V=\mathbb{R} \times M$ by $i^{+}$(resp. $i^{-}$), one can write (see [7, Sect. 6.2]):

$$
\begin{aligned}
\hat{V}_{\tilde{\alpha}} & B_{\tilde{\alpha}}^{+}(M) \cup\{\infty\}=\left(\mathbb{R} \times M_{B, \tilde{\alpha}}^{+}\right) \cup\left\{i^{+}\right\} \\
& =\left(\mathbb{R} \times\left(M \cup \partial_{B, \tilde{\alpha}}^{+} M\right)\right) \cup\left\{i^{+}\right\} \\
& =V \cup\left(\mathbb{R} \times \partial_{B, \tilde{\alpha}}^{+} M\right) \cup\left\{i^{+}\right\} \\
& =V \cup\left(\mathbb{R} \times \partial_{C}^{+} M\right) \cup\left(\mathbb{R} \times \partial_{\mathcal{B}, \tilde{\alpha}}^{+} M\right) \cup\left\{i^{+}\right\} . \\
\hat{\partial}_{\tilde{\alpha}} V & \equiv\left(\mathbb{R} \times \partial_{B, \tilde{\alpha}}^{+} M\right) \cup\left\{i^{+}\right\}=\left(\mathbb{R} \times \partial_{C}^{+} M\right) \cup\left(\mathbb{R} \times \partial_{\mathcal{B}, \tilde{\alpha}}^{+} M\right) \cup\left\{i^{+}\right\} . \\
\check{V}_{\tilde{\alpha}} & \equiv V \cup\left(\mathbb{R} \times \partial_{C}^{-} M\right) \cup\left(\mathbb{R} \times \partial_{\mathcal{B}, \tilde{\alpha}}^{-} M\right) \cup\left\{i^{-}\right\} . \\
\check{\partial}_{\tilde{\alpha}} V & \equiv\left(\mathbb{R} \times \partial_{C}^{-} M\right) \cup\left(\mathbb{R} \times \partial_{\mathcal{B}, \tilde{\alpha}}^{-} M\right) \cup\left\{i^{-}\right\} .
\end{aligned}
$$

\subsection{Topology and S-relations}

The future and past chronological topologies can be characterized by using the identifications (3.13) in terms of the following limit operators, whose dependence on $\tilde{\alpha}$, via their domain, is implicitly assumed: given a sequence $\left\{f_{n}\right\}_{n}$ and an element $f$ in $B_{\tilde{\alpha}}^{ \pm}(M) \cup\{ \pm \infty\} 2^{2}$

$$
\begin{aligned}
& f \in \hat{L}\left(\left\{f_{n}\right\}\right) \Longleftrightarrow\left\{\begin{array}{l}
(a) f \leqslant \liminf _{n} f_{n} \text { and } \\
(b) \forall g \in B_{\tilde{\alpha}}^{+}(M) \text { with } f \leqslant g \leqslant \limsup _{n} f_{n}, \text { it is } g=f
\end{array}\right. \\
& f \in \check{L}\left(\left\{f_{n}\right\}\right) \Longleftrightarrow\left\{\begin{array}{l}
(a) f \geq \limsup _{n} f_{n} \text { and } \\
(b) \forall g \in B_{\tilde{\alpha}}^{-}(M) \text { with } f \geq g \geq \liminf _{n} f_{n}, \text { it is } g=f .
\end{array}\right.
\end{aligned}
$$

The future and past chronological topologies are coarser than the pointwise topology in the space of Busemann functions. In fact, we have the following adaptation of [7, Proposition 5.29].

Proposition 3.2 Consider a sequence $\left\{f_{n}\right\} \subset B_{\bar{\alpha}}^{ \pm}(M)$ which converges pointwise to a function $f \in B_{\bar{\alpha}}^{ \pm}(M)$. Then, $f$ is the unique future (resp. past) chronological limit of $\left\{f_{n}\right\}$.

In particular, if $f_{n}=d_{\left(\Omega_{\bar{\alpha}}^{n}, x^{n}\right)}^{ \pm}$with $\Omega_{\bar{\alpha}}^{n} \rightarrow \Omega_{\bar{\alpha}}(\in \mathbb{R})$ and $x^{n} \rightarrow x\left(\in M_{C}^{ \pm}\right)$, then $f=d_{\left(\Omega_{\bar{\alpha}}, x\right)}^{+} \in \hat{L}\left(f_{n}\right)$ (resp. $\left.f=d_{\left(\Omega_{\bar{\alpha}}, x\right)}^{-} \in \check{L}\left(f_{n}\right)\right)$ is the unique future (resp. past) chronological limit of $\left\{f_{n}\right\}$.

In order to study the S-relations (2.1), (2.2) between terminal sets, their common pasts and futures must be considered. If $P_{\tilde{\alpha}}$ is generated by a future-directed timelike curve $\gamma(t)=(t, c(t)), t \in[a, \infty)$, then $\uparrow_{\tilde{\alpha}} P_{\tilde{\alpha}}=\emptyset$, and so, $P_{\tilde{\alpha}}$ is S-related only to $\emptyset$. Analogously, if $F_{\tilde{\alpha}}$ is generated by a past-directed timelike curve $\gamma(t)=$

\footnotetext{
${ }^{2}$ The term $\{ \pm \infty\}$ is included here in order to obtain a more direct relation with the c-boundary of spacetimes. Note that this term is not necessary when one works with Busemann compactifications, as a quotient space by additive constants is considered then (compare with [7).
} 
$(-t, c(t)), t \in[-a, \infty)$, then $\downarrow_{\tilde{\alpha}} F_{\tilde{\alpha}}=\emptyset$, and $F_{\tilde{\alpha}}$ is S-related only to $\emptyset$. If, instead, $\pm \Omega<\infty$, then $b_{c, \tilde{\alpha}}^{ \pm}=d_{p^{ \pm}}^{ \pm}$, with $p^{ \pm}=\left(\Omega_{\tilde{\alpha}}, x^{ \pm}\right)$(recall $\left.3.9 p\right)$ and, thus:

$$
\begin{aligned}
& \uparrow P_{\tilde{\alpha}}=\uparrow P_{\tilde{\alpha}}\left(d_{p^{+}}^{+}\right)=F_{\tilde{\alpha}}\left(d_{p^{+}}^{-}\right) \\
& \downarrow F_{\tilde{\alpha}}=\downarrow F_{\tilde{\alpha}}\left(d_{p^{-}}^{-}\right)=P_{\tilde{\alpha}}\left(d_{p^{-}}^{+}\right) .
\end{aligned}
$$

For the first equality of each line in 3.14 recall Section 3.1 and for the second one recall [7, Lemma 6.11].

Remark 3.3 Previous expressions allow to deduce the following facts: (i) if $p=$ $p^{+}=p^{-}=\left(\Omega_{\tilde{\alpha}}, x^{s}\right) \in \mathbb{R} \times \partial_{C}^{s} M$ (i.e., the future-directed and past-directed timelike curves generating the terminal sets end at the same point of $\mathbb{R} \times \partial_{C}^{s} M$, recall (2.5)), then $\uparrow P_{\tilde{\alpha}}\left(d_{p}^{+}\right)=F_{\tilde{\alpha}}\left(d_{p}^{-}\right), \downarrow F_{\tilde{\alpha}}\left(d_{p}^{-}\right)=P_{\tilde{\alpha}}\left(d_{p}^{+}\right)$, and so, $P_{\tilde{\alpha}}\left(d_{p}^{+}\right) \sim_{S} F_{\tilde{\alpha}}\left(d_{p}^{-}\right)$; (ii) if $d_{Q}^{+}$is not a generalized distance, but $\left(M, d^{+}\right)$has an evenly pairing boundary (see [7. Def. 3.34]), then $d_{p^{ \pm}}^{\mp}=\Omega_{\tilde{\alpha}} \pm d^{\mp}\left(\cdot, x^{ \pm}\right)= \pm \infty$ whenever $x^{ \pm} \in \partial_{C}^{ \pm} M \backslash \partial_{C}^{s} M$ and, in this case, $\uparrow P_{\tilde{\alpha}}\left(d_{p^{+}}^{+}\right)=\emptyset=\downarrow F_{\tilde{\alpha}}\left(d_{p^{-}}^{-}\right)$(for non evenly pairing boundaries, the situation is much subtler, see [7, Th $1.2(1 \mathrm{~B})(\mathrm{c} 2)])$.

\subsection{Main result}

The action (3.10) defined on Busemann functions (always under condition (3.2)) induces also a natural action on $S$-related pairs, each one generating the following orbit (line):

$$
\begin{aligned}
& \mathcal{L}\left(P_{\tilde{\alpha}}, \emptyset\right):=\left\{\left(P_{\tilde{\alpha}}^{\prime}, \emptyset\right): P_{\tilde{\alpha}}^{\prime}=P_{\tilde{\alpha}}\left(b_{c, \alpha}^{+}+k\right), k \in \mathbb{R}\right\} \text { where } P_{\tilde{\alpha}}=P_{\tilde{\alpha}}\left(b_{c, \tilde{\alpha}}^{+}\right) \\
& \mathcal{L}\left(\emptyset, F_{\tilde{\alpha}}\right):=\left\{\left(\emptyset, F_{\tilde{\alpha}}^{\prime}\right): F_{\tilde{\alpha}}^{\prime}=F_{\tilde{\alpha}}\left(b_{c, \tilde{\alpha}}^{-}+k\right), k \in \mathbb{R}\right\} \text { where } F_{\tilde{\alpha}}=F_{\tilde{\alpha}}\left(b_{c, \tilde{\alpha}}^{-}\right)
\end{aligned}
$$

and

$$
\mathcal{L}\left(P_{\tilde{\alpha}}, F_{\tilde{\alpha}}\right):=\left\{\left(P_{\tilde{\alpha}}^{\prime}, F_{\tilde{\alpha}}^{\prime}\right): P_{\tilde{\alpha}}^{\prime}=P_{\tilde{\alpha}}\left(d_{p}^{+}+k\right), F_{\tilde{\alpha}}^{\prime}=F_{\tilde{\alpha}}\left(d_{p^{\prime}}^{-}+k\right), k \in \mathbb{R}\right\},
$$

where $P_{\tilde{\alpha}}=P_{\tilde{\alpha}}\left(d_{p}^{+}\right)$and $F_{\tilde{\alpha}}=F_{\tilde{\alpha}}\left(d_{p^{\prime}}^{-}\right)$. Now, Theorem 1.2 in [7] yields, in particular, the following result.

Theorem 3.4 Let $V_{\tilde{\alpha}}=\left(\mathbb{R} \times M, g_{\tilde{\alpha}}\right)$ be a spacetime as in (3.1) satisfying the overall condition (3.2). If $d_{Q}^{+}$is a generalized distance, then the c-boundary $\partial_{\tilde{\alpha}} V$ has the following structures.

Point Set:

- The future (resp. past) c-boundary $\hat{\partial}_{\tilde{\alpha}} V$ (resp. $\check{\partial}_{\tilde{\alpha}} V$ ) is naturally a point set cone with base $\partial_{B, \tilde{\alpha}}^{+} M$ (resp. $\left.\partial_{B, \tilde{\alpha}}^{-} M\right)$ and apex $i^{+}$(resp. $\left.i^{-}\right)$.

- The total c-boundary $\partial_{\tilde{\alpha}} V$ is the quotient set of the partial boundaries $\hat{\partial}_{\tilde{\alpha}} V$, $\check{\partial}_{\tilde{\alpha}} V$ under the $S$-relation. So, it is composed by the set of all the lines $\mathcal{L}\left(\left(P_{\tilde{\alpha}}\left(d_{p}^{+}\right), F_{\tilde{\alpha}}\left(d_{p}^{-}\right)\right)\right), \mathcal{L}\left(\left(P_{\tilde{\alpha}}\left(b_{c, \tilde{\alpha}}^{+}\right), \emptyset\right)\right), \mathcal{L}\left(\left(\emptyset, F_{\tilde{\alpha}}\left(b_{c, \tilde{\alpha}}^{-}\right)\right)\right)$, with $p \in \mathbb{R} \times \partial_{C}^{s} M$, $b_{c, \tilde{\alpha}}^{ \pm} \in \mathcal{B}_{\tilde{\alpha}}^{ \pm}(M)$. 
Causality:

- The lines $\mathcal{L}\left(\left(P_{\tilde{\alpha}}\left(d_{p}^{+}\right), F_{\tilde{\alpha}}\left(d_{p}^{-}\right)\right)\right)$, with $p \in \mathbb{R} \times \partial_{C}^{s} M$, are timelike, i.e. any two points of the line are chronologically related.

- The lines $\mathcal{L}\left(\left(P_{\tilde{\alpha}}\left(b_{c \tilde{\alpha}}^{+}\right), \emptyset\right)\right), \mathcal{L}\left(\left(\emptyset, F_{\tilde{\alpha}}\left(b_{c, \tilde{\alpha}}^{-}\right)\right)\right.$, with $b_{c, \tilde{\alpha}}^{ \pm} \in \mathcal{B}_{\tilde{\alpha}}^{ \pm}(M)$, are horismotic, i.e. any two points of the lines are causally but not chronologically related.

Topology:

- If $M_{B, \tilde{\alpha}}^{+}$(resp. $\left.M_{B, \tilde{\alpha}}^{-}\right)$is Hausdorff, the future (resp. past) c-boundary has the structure of a (topological) cone with base $\partial_{B, \tilde{\alpha}}^{+} M$ (resp. $\left.\partial_{B, \tilde{\alpha}}^{-} M\right)$ and apex $i^{+}$ (resp. $\left.i^{-}\right)$.

- If $M_{C, \tilde{\alpha}}^{s}$ is locally compact then $\bar{V}_{\tilde{\alpha}}$ is (topologically) simple, that is, each TIP (resp. each TIF) determines a unique pair in $\partial_{\tilde{\alpha}} V$ and $(P, F) \in L\left(\left\{\left(P_{n}, F_{n}\right)\right\}\right)$ when either $P \in \hat{L}\left(\left\{P_{n}\right\}\right)$ or $F \in \check{L}\left(\left\{F_{n}\right\}\right)$. Equivalently, $\bar{V}_{\tilde{\alpha}}$ coincides with the quotient topological space of the future and past c-completions $\hat{V}_{\tilde{\alpha}}$ and $\check{V}_{\tilde{\alpha}}$ under the S-relation.

\section{Framework for isocausal comparison}

\subsection{Causal mappings and c-boundary}

Let us begin by recalling the notion of isocausality (see [10] for further details):

Definition 4.1 Let $V \equiv(V, g), V^{\prime} \equiv\left(V^{\prime}, g^{\prime}\right)$ be two spacetimes. A diffeomorphism $\Phi: V \rightarrow V^{\prime}$ is called a causal mapping if all the future causal vectors of $V$ are mapped by the differerential $d \Phi$ into future causal vectors of $V^{\prime}$. In this case, we write $V \prec_{\Phi} V^{\prime}$. $V$ is causally related to $V^{\prime}$, denoted $V \prec V^{\prime}$, if $V \prec_{\Phi} V^{\prime}$ for some causal mapping $\Phi$; moreover, $V$ is isocausal to $V^{\prime}$ if $V \prec V^{\prime}$ and $V^{\prime} \prec V$.

In particular, when the underlying manifolds coincide and the identity Id $: V \rightarrow$ $V^{\prime}$ is a causal mapping (namely, $g(v, v) \leqslant 0$ implies $g^{\prime}(v, v) \leqslant 0$ ) we say that the cones of $g^{\prime}$ are wider than those of $g$ and write $V \prec_{0} V^{\prime}$ or, simply, $g \prec_{0} g^{\prime}$.

Remark 4.2 Obviously, $V \prec_{\Phi} V^{\prime}$ plus $V^{\prime} \prec_{\Phi}^{-1} V$ imply not only that $V$ and $V^{\prime}$ are isocausal but also that $\Phi$ is a conformal map; so, $g$ and $g^{\prime}$ are conformal metrics. If $g \prec_{0} g^{\prime}$ and $g^{\prime} \prec_{0} g$ then $g$ and $g^{\prime}$ are pointwise conformal metrics.

Typically, we will take three Lorentzian metrics $g, g^{\prime}, g^{\prime \prime}$ on the same manifold which satisfy $g \prec_{0} g^{\prime} \prec_{0} g^{\prime \prime}$ and use the following obvious result: if $g$ and $g^{\prime \prime}$ are conformal then $g$ and $g^{\prime}$ (and $g^{\prime \prime}$ ) are isocausal.

Remark 4.3 Let us discuss the relation between the c-boundary points of two causally related spacetimes; by taking pull-back metrics we can consider the case $g \prec_{0} g^{\prime}$ with no loss of generality. 
If $P$ is a TIP for $g$, then $I_{g^{\prime}}^{-}(P)$ is also a TIP for $g^{\prime}$ (write $P=I_{g}^{-}(\gamma)$ and notice $\left.I_{g^{\prime}}^{-}(P)=I_{g^{\prime}}^{-}(\gamma)\right)$. This may suggest a procedure to include the c-boundary for $g$ into the c-boundary for $g^{\prime}$, i.e. consider the map $P \mapsto I_{g^{\prime}}^{-}(P)$. However, this procedure is not satisfactory in general, as there may exist two different TIPs $P_{1} \neq P_{2}$ for $g$ such that $I_{g^{\prime}}^{-}\left(P_{1}\right)=I_{g^{\prime}}^{-}\left(P_{2}\right)$ (violating the injectivity of the inclusion). This undesirable property may happen even if $g$ and $g^{\prime}$ are isocausal (see Example 5.7).

However, as we will see in the following sections, there are certain conditions under which this procedure succeed.

\subsection{Spacetimes isocausal to standard stationary ones}

Our aim is to study the c-boundary of the spacetime

$$
V=(\mathbb{R} \times M, g), \quad g=-d t^{2}+\omega_{t} \otimes d t+d t \otimes \omega_{t}+h_{t},
$$

where $\omega_{t}$ is a one form on $\mathbb{R} \times M$ such that $\omega_{t}(\partial / \partial t)=0$ (when necessary, and abusing of notation, it will be regarded as a one form on each slice $\{t\} \times M)$. This will be carried out by comparing it with the c-boundaries of the spacetimes

$$
\begin{aligned}
& V_{c l}=\left(\mathbb{R} \times M, g_{c l}\right), \quad g_{c l}\left(\equiv g_{\tilde{\alpha}=1}\right)=-d t^{2}+\omega \otimes d t+d t \otimes \omega+h \\
& V_{o p}=\left(\mathbb{R} \times M, g_{o p}\right), \quad g_{o p}\left(\equiv g_{\tilde{\alpha}=\alpha}\right)=-d t^{2}+\alpha(t) \omega \otimes d t+\alpha(t) d t \otimes \omega+\alpha^{2}(t) h,
\end{aligned}
$$

where one implicitly assumes the relations

$$
g_{c l} \prec_{0} g \prec_{0} g_{o p} .
$$

First, it is convenient to rewrite condition (4.3) in terms of the corresponding metric coefficients. To this aim, let $\gamma(s)=(t(s), c(s))$ be a future/past-directed timelike curve for $g$. Then:

$$
-\dot{t}^{2}+2 \omega_{t}(\dot{c}) \dot{t}+h_{t}(\dot{c}, \dot{c})<0 .
$$

Solving this second-order equation in $\dot{t}$, and taking into account that $\pm \dot{t}>0$, one directly deduces:

$$
\pm \dot{t}>F_{t}^{ \pm}(\dot{c}) \quad \text { with } \quad F_{t}^{ \pm}(\dot{c}):=\sqrt{\omega_{t}^{2}(\dot{c})+h_{t}(\dot{c}, \dot{c})} \pm \omega_{t}(\dot{c}) .
$$

So, the timelike character of $\gamma$ for the metric $g$ is determined by the functions $F_{t}^{ \pm}$. Reasoning analogously, if $\gamma(s)=(t(s), c(s))$ is a future/past-directed timelike curve for $g_{c l}$ (resp. $\left.g_{o p}\right)$ then

$\pm \dot{t}>F^{ \pm}(\dot{c}) \quad\left(\right.$ resp. $\left.\pm \dot{t}>\alpha(t) F^{ \pm}(\dot{c})\right) \quad$ with $F^{ \pm}(\dot{c}):=\sqrt{\omega^{2}(\dot{c})+h(\dot{c}, \dot{c})} \pm \omega(\dot{c})$.

Remark 4.4 Summarizing, the causal character of the future/past directed curve $\gamma(s)=(t(s), c(s))$ for $g, g_{c l}$ or $g_{o p}$, is characterized as follows:

$\begin{array}{llrl}\gamma \text { is timelike (causal; lightlike) for } g & \text { if } & F_{t}^{ \pm}(\dot{c}) & <(\leqslant ;=) \pm \dot{t} \\ \gamma \text { is timelike (causal; lightlike) for } g_{c l} & \text { if } & F^{ \pm}(\dot{c}) & <(\leqslant ;=) \pm \dot{t} \\ \gamma \text { is timelike (causal; lightlike) for } g_{o p} & \text { if } & \alpha(t) F^{ \pm}(\dot{c}) & <(\leqslant ;=) \pm \dot{t}\end{array}$ 
In conclusion, applying (4.4) to tangent vectors, one deduces directly:

Proposition 4.5 The relations $g_{c l} \prec_{0} g \prec_{0} g_{o p}$ hold iff the following inequalities hold:

$$
\alpha(t) F^{ \pm}(\cdot) \leqslant F_{t}^{ \pm}(\cdot) \leqslant F^{ \pm}(\cdot) .
$$

On the other hand:

Proposition $4.6 g_{o p}$ is conformal to $g_{c l}$ iff (3.2) holds for $\tilde{\alpha}=\alpha$.

Proof. For the implication to the right, assume, for example,

$$
\int_{0}^{\infty} \frac{d s}{\alpha(s)}<\infty
$$

From Proposition 3.1 with $\tilde{\alpha} \equiv \alpha$, no timelike curve $\gamma$ for $g_{o p}$ satisfies $I_{o p}^{-}(\gamma)=V$, as there will always exist some point with temporal component close enough to $\Omega$ (the upper limit of the temporal component of $\gamma$ ) which does not belong to the chronological past of $\gamma$. Hence, $i^{+} \equiv V$ does not belong to the future c-boundary of $V_{o p}$. Nevertheless, it is easy to check that $i^{+}$belongs to the future c-boundary of $V_{c l}$ (see [7] or recall that $i^{+}$is the past of any curve type $\left.t \mapsto\left(t, x_{0}\right)\right)$. Therefore, the future c-boundaries of $V_{o p}$ and $V_{c l}$ cannot be identified, and so, $g_{o p}, g_{c l}$ are not conformally related.

For the implication to the left, note that, under 3.2 with $\tilde{\alpha}=\alpha$, the change of variables $d s=d t / \alpha(t)$ takes $V_{o p}$ into a spacetime conformal to $V_{c l}$.

Corollary 4.7 If the relations $g_{c l} \prec_{0} g \prec_{0} g_{o p}$ hold, then $g_{c l}$ and $g_{o p}$ are conformal, and thus, $g$ is isocausal to them.

Proof. From Proposition $4.5, \alpha(t) F^{ \pm}(\cdot) \leqslant F^{ \pm}(\cdot)$. Thus, $\alpha(t) \leqslant 1$ and:

$$
\int_{0}^{\infty} \frac{d s}{\alpha(s)} \geq \int_{0}^{\infty} 1 d s=\infty, \quad \int_{-\infty}^{0} \frac{d s}{\alpha(s)} \geq \int_{-\infty}^{0} 1 d s=\infty
$$

that is, condition (3.2) holds for $\tilde{\alpha}=\alpha$. Therefore, from Proposition 4.6, $g_{o p}$ is conformal to $g_{c l}$. The last assertion is straightforward from Remark 4.2 .

Remark 4.8 Observe that all the constructions provided in Section $3\left(B_{\tilde{\alpha}}^{ \pm}(M)\right.$, $\left.M_{B, \tilde{\alpha}}^{+} \ldots\right)$ can be developed for both, $\tilde{\alpha}=\alpha$ and $\tilde{\alpha}=1$. Moreover, when $g_{o p}$ and $g_{c l}$ are conformal (in particular, under condition (4.3)), such constructions are equivalent. In these cases, we will denote such constructions with no mention to $\tilde{\alpha}$.

\section{Partial boundaries $\hat{\partial} V, \partial \check{V}$ through isocausality}

From now on, $V=(\mathbb{R} \times M, g)$ will denote a spacetime as in 4.1$)$ which satisfies condition (4.3) for the metrics in 4.2 (unless other hypotheses are explicitly assumed), so that Corollary 4.7 is applicable. In particular, the inequality $\alpha \leqslant 1$ holds (Proposition 4.5). 


\subsection{Information on $\hat{\partial} V$ at the point set level}

Along this section, we will use repeatedly the following set (see $(3.4)$ and $(3.5)$ ):

$$
P_{\tilde{\alpha}}\left(b_{c, \tilde{\alpha}}^{+}\right)=\left\{\left(t_{0}, x_{0}\right) \in V: \int_{0}^{t_{0}} \frac{d s}{\tilde{\alpha}(s)}<b_{c, \tilde{\alpha}}^{+}\left(x_{0}\right)\right\} .
$$

Convention 5.1 The objects associated to the metric $g$ (resp. $g_{o p} ; g_{c l}$ ) present no subindex (resp. subindex op; subindex cl). We will focus on the future c-boundary $\hat{\partial} V$ for $(V, g)$ (by comparing with $\hat{\partial}_{c l} V \equiv \hat{\partial}_{o p} V$ ), being the arguments and results for the past c-boundaries $\check{\partial} V, \check{\partial}_{c l} V \equiv \check{\partial}_{o p} V$ totally analogous (see Subsection 5.4 ).

As suggested in Remark 4.3 , we are going to define a map which takes $\hat{V}_{c l}$ into $\hat{V}$ by mapping every TIP $P_{c l}$ for $g_{c l}$ into the TIP $I^{-}\left(P_{c l}\right)$ for $g$. In order to establish under which conditions this map works, we need to establish previously some technical results:

Proposition 5.2 If $\gamma:[a, \infty) \rightarrow V, \gamma(t)=(t, c(t))$ is a future-directed timelike curve for $g$ such that $c(t)$ remains in a bounded region of $\left(M, d^{+}\right)$then $I^{-}(\gamma)=V$.

Proof. Take any $\left(t_{0}, x_{0}\right) \in V$. From the hypothesis, there exists $K>0$ such that $d^{+}\left(x_{0}, c(t)\right)<K$ for all $t \in[a, \infty)$. In particular,

$$
d^{+}\left(x_{0}, c(t)\right)<K<\lim _{t \nearrow \infty} \int_{t_{0}}^{t} d s=\infty .
$$

Therefore, from (5.2) and Proposition 3.1 with $\tilde{\alpha} \equiv 1$, one has $\left(t_{0}, x_{0}\right) \ll_{c l}(t, c(t))$ for $t$ big enough, thus $V=I_{c l}^{-}(\gamma) \subset I^{-}(\gamma)$ (the last inclusion because $g_{c l} \prec_{0} g$ ).

Proposition 5.3 Let $V$ be a spacetime as in 4.1), with $g_{c l}, g_{o p}$ as in (4.2), and satisfying 4.3.). Under the integral condition

$$
\int_{0}^{\infty}\left(\frac{1}{\alpha(s)}-1\right) d s<\infty
$$

the following assertions hold:

(1) Let $P_{c l}=P_{c l}\left(b_{c, c l}^{+}\right)\left(\right.$recall 3.5) ) with $b_{c, c l}^{+} \in B_{c l}^{+}(M)$ and $c:[a, \Omega) \rightarrow M$, and put:

$$
K_{\Omega}=\int_{0}^{\Omega}\left(\frac{1}{\alpha(s)}-1\right) d s .
$$

Then, $b_{c, c l}^{+}+K_{\Omega} \in B_{o p}^{+}(M)$ and $I_{o p}^{-}\left(P_{c l}\right)=P_{o p}\left(b_{c, c l}^{+}+K_{\Omega}\right)$.

(2) If $P_{c l}^{1} \subsetneq P_{c l}^{2}$ then $I_{o p}^{-}\left(P_{c l}^{1}\right) \subsetneq I_{o p}^{-}\left(P_{c l}^{2}\right)$. Moreover, if $P_{c l}^{1} \neq P_{c l}^{2}$ then $I_{o p}^{-}\left(P_{c l}^{1}\right) \neq$ $I_{o p}^{-}\left(P_{c l}^{2}\right)$. 
Proof. (1) Consider the curve $\gamma(t)=(t, c(t))$, with $t \in[a, \Omega)$, which is timelike for $g_{c l}$ and satisfies $I_{c l}^{-}(\gamma)=P_{c l}$. So, $I_{o p}^{-}\left(P_{c l}\right)=I_{o p}^{-}(\gamma)=P_{o p}\left(b_{c, o p}^{+}\right)$where $b_{c, o p}^{+}$is defined by (3.4) with $\tilde{\alpha}=\alpha$ (recall Convention 5.1). But,

$$
\begin{aligned}
b_{c, o p}^{+}(\cdot) & =\lim _{t \nearrow \Omega}\left(\int_{0}^{t}\left(\frac{1}{\alpha(s)}-1\right) d s+t-d^{+}(\cdot, c(t))\right) \\
& =K_{\Omega}+b_{c, c l}^{+}(\cdot) .
\end{aligned}
$$

Hence, $I_{o p}^{-}\left(P_{c l}\right)=P_{o p}\left(b_{c, c l}^{+}+K_{\Omega}\right)$ with $b_{c, c l}^{+}+K_{\Omega} \in B_{o p}^{+}(M)$ (notice that 5.3 ) implies $\left.K_{\Omega}<\infty\right)$.

(2) Consider $c_{i}:\left[a_{i}, \Omega_{i}\right) \rightarrow M$ such that $P_{c l}^{i}=P_{c l}\left(b_{c_{i}, c l}^{+}\right)$. From the inclusion $P_{c l}^{1} \subsetneq P_{c l}^{2}$, we deduce that $b_{c_{1}, c l}^{+} \leqslant b_{c_{2}, c l}^{+}$with strict inequality at some point. Moreover, $\Omega_{1} \leqslant \Omega_{2}$. In fact, $d^{+}\left(c_{1}(s), c_{1}(t)\right)<t-s$ for all $t, s \in\left[a_{1}, \Omega_{1}\right)$ with $s<t$ and, then (recall that $t-d^{+}\left(p, c_{i}(t)\right)$ is non-decreasing with $t$ ),

$s<t-d^{+}\left(c_{1}(s), c_{1}(t)\right) \leqslant \lim _{t \nearrow \Omega_{1}}\left(t-d^{+}\left(c_{1}(s), c_{1}(t)\right)\right)=b_{c_{1}, c l}^{+}\left(c_{1}(s)\right) \leqslant b_{c_{2}, c l}^{+}\left(c_{1}(s)\right) \leqslant \Omega_{2}$,

for all $s \in\left[a_{1}, \Omega_{1}\right)$, and thus, $\Omega_{1} \leqslant \Omega_{2}$ as required. Therefore, $b_{c_{1}, c l}^{+}+K_{\Omega_{1}} \leqslant$ $b_{c_{2}, c l}^{+}+K_{\Omega_{2}}$ with strict inequality at some point. Taking into account that the left and right members of the inequality characterize $P_{o p}^{1}$ and $P_{o p}^{2}$, resp., it directly follows $P_{o p}^{1} \subsetneq P_{o p}^{2}$.

For the last assertion, assume by contradiction that $P_{c l}^{1} \neq P_{c l}^{2}$ but

$$
I_{o p}^{-}\left(P_{c l}^{1}\right)=I_{o p}^{-}\left(P_{c l}^{2}\right) \text {. }
$$

From part (1), we deduce that $K_{\Omega_{1}}+b_{c_{1}, c l}^{+}=K_{\Omega_{2}}+b_{c_{2}, c l}^{+}$. Suppose that $K_{\Omega_{2}} \leqslant K_{\Omega_{1}}$ (the case $K_{\Omega_{2}} \geq K_{\Omega_{1}}$ is analogous) and observe that, as $b_{c_{2}, c l}^{+}=b_{c_{1}, c l}^{+}+\left(K_{\Omega_{1}}-K_{\Omega_{2}}\right)$, necessarily $P_{c l}^{1}=P_{c l}\left(b_{c_{1}, c l}^{+}\right) \subset P_{c l}\left(b_{c_{2}, c l}^{+}\right)=P_{c l}^{2}$. From the first part of (2), we deduce $I_{o p}^{-}\left(P_{c l}^{1}\right) \varsubsetneqq I_{o p}^{-}\left(P_{c l}^{2}\right)$, in contradiction to 5.4.

Remark 5.4 In the proof of Proposition 5.3 condition (5.3) (namely, the causal cones of $g$ and $g_{c l}$ approach at $t$-infinity) is only needed when the IPs involved are of the form $P_{c l}=P_{c l}\left(b_{c, c l}^{+}\right)$with $\Omega=\infty$, i.e. when $P_{c l} \notin \mathbb{R} \times M_{C}^{+}$(recall $\left.3.13 p\right)$. In fact, if $P_{c l} \in \mathbb{R} \times M_{C}^{+}$then the change $b_{c, c l}^{+} \mapsto b_{c, o p}^{+}=b_{c, c l}^{+}+K_{\Omega}$ is just a re-scaling requested by (5.1). If (5.3) does not hold then $K_{\Omega}=\infty$, and our procedure takes every $P_{c l} \notin \mathbb{R} \times M_{C}^{+}$into $I_{o p}^{-}\left(P_{c l}\right) \equiv V \equiv i^{+}$, being not one to one.

On the other hand, in the same proof condition 4.3 is only needed to ensure that inequality $\alpha \leqslant 1$ holds.

Theorem 5.5 Let $V=(\mathbb{R} \times M, g)$ be a spacetime as in 4.1) such that $g_{c l} \prec_{0} g \prec_{0}$ $g_{\text {op }}$ holds. If (5.3) holds, then the map

$$
\begin{array}{cccc}
\hat{j}: & \hat{V}_{c l} & \rightarrow & \hat{V} \\
P_{c l} & \mapsto & I^{-}\left(P_{c l}\right)
\end{array}
$$

is injective. So, $\hat{V}$ contains a point set cone of base $M_{B}^{+}$and apex $i^{+}$. 
Proof. Consider $P_{c l}^{1}=P_{c l}\left(b_{c_{1}, c l}^{+}\right), P_{c l}^{2}=P_{c l}\left(b_{c_{2}, c l}^{+}\right)$, with $c_{i}:\left[a_{i}, \Omega_{i}\right) \rightarrow M$. Suppose that $\hat{j}\left(P_{c l}^{1}\right)=I^{-}\left(P_{c l}^{1}\right)=I^{-}\left(P_{c l}^{2}\right)=\hat{j}\left(P_{c l}^{2}\right)$. As $g \prec_{0} g_{o p}$, necessarily $I_{o p}^{-}\left(P_{c l}^{1}\right)=$ $I_{o p}^{-}\left(P_{c l}^{2}\right)$, and Proposition $5.3(2)$ ensures that $P_{c l}^{1}=P_{c l}^{2}$. For the last assertion, recall that from 3.13$) \hat{V}_{c l} \equiv\left(\mathbb{R} \times M_{B}^{+}\right) \cup\left\{i^{+}\right\}$.

Finally, we can particularize Theorem 5.5 to some relevant cases:

Corollary 5.6 Let $V=(\mathbb{R} \times M, g)$ be a spacetime as in (4.1) such that $g_{c l} \prec_{0} g \prec_{0}$ $g_{\text {op }}$.

(1) If $\left(M, d^{+}\right)$is bounded, then $\partial_{\mathcal{B}}^{+} M=\emptyset$ and $\hat{\partial} V$ contains a cone of base $\partial_{C}^{+} M$ and apex $i^{+}$. If, in addition, $\left(M, d^{+}\right)$is complete (thus, compact), then $\hat{\partial} V$ coincides with $i^{+}$.

(2) If $\left(M, d^{+}\right)$is complete and (5.3) holds, then $\partial_{C}^{+} M=\emptyset$ and $\hat{\partial} V$ contains a cone of base $\partial_{\mathcal{B}}^{+} M$ and apex $i^{+}$(recall Remark 4.8).

Proof. (1) For the first assertion, as boundedness implies $\partial_{\mathcal{B}}^{+} M=\emptyset$, the result follows from $\mathrm{Th}$. 5.5. For the second one, note that any inextendible timelike curve $\gamma:[a, \Omega) \rightarrow V, \gamma(t)=(t, c(t))$ must satisfy $\Omega=\infty$ : in fact, otherwise, Proposition 3.1 implies length $(c)<\infty$, and thus, $c(t) \rightarrow x^{+} \in \partial_{C}^{+} M$, in contradiction to the fact that $\partial_{C}^{+} M=\emptyset$. Therefore, from Proposition 5.2, the past of any inextendible future-directed timelike curve $\gamma$ is $V$.

(2) As completeness implies $\partial_{C}^{+} M$ is empty, the result follows again by using Th.

\section{5 .}

\subsection{Discussion}

The following examples and comments show the optimality of the hypotheses and results in previous subsection. The ambient hypotheses $g_{c l} \prec_{0} g \prec_{0} g_{o p}$ will not be discussed further, as it becomes natural for isocausal comparison (recall Corollary 4.7) and imply $\alpha \leqslant 1$ from Proposition 4.5. So, let us start with inequality (5.3). As commented in Remark 5.4 , this inequality is necessary to ensure that $\hat{j}$ is one to one. This is illustrated in the following trivial example.

Example 5.7 Consider the following metrics on $V=\mathbb{R} \times \mathbb{R}$ :

$$
g_{c l}=-d t^{2}+d x^{2}, \quad g=-d t^{2}+d x^{2} / 2, \quad g_{o p}=-d t^{2}+d x^{2} / 3 .
$$

These metrics (all of them isometric) satisfy $g_{c l} \prec_{0} g \prec_{0} g_{o p}$, but condition 5.3p does not hold. Then, any TIP $P_{c l}$ for $g_{c l}$, defined by a lightlike curvt ${ }^{3}$ of the form $\gamma(t)=(t, \pm t)$, satisfies $I^{-}\left(P_{c l}\right)=V$, being $\hat{j}\left(\hat{\partial}_{c l} V\right)=i^{+}$(recall Remark 5.4).

\footnotetext{
${ }^{3}$ Here and in what follows, recall that the chronological past of any inextensible future-directed lightlike curve is also a TIP (notice that the converse also holds, see [8, Sect 3.3]).
} 
Remark 5.8 Even though condition (5.3) in Theorem 5.5]solves the problem pointed out in Remark 4.3 , the following question arises now. From the definition of $\hat{j}$ and the relations $g_{c l} \prec_{0} g \prec_{0} g_{o p}$, the following relations hold:

$$
P_{c l} \subset \hat{j}\left(P_{c l}\right), \quad I_{o p}^{-}\left(\hat{j}\left(P_{c l}\right)\right)=I_{o p}^{-}\left(P_{c l}\right) .
$$

However, $\hat{j}\left(P_{c l}\right)$ may not be the unique TIP for $g$ satisfying these relations. As we will see next, this property is closely related to the lack of surjectivity or continuity for $\hat{j}$ in general.

The explicit example constructed in [5] provides such a non-surjective and noncontinuous man $\hat{j}^{4} \hat{j}$. In that example the contribution to the future c-boundary $\hat{\partial}_{c l} V$ comes completely from the forward Cauchy boundary $\partial_{C}^{+} M$, i.e., $\hat{\partial}_{c l} V=$ $\left(\mathbb{R} \times \partial_{C}^{+} M\right) \cup\left\{i^{+}\right\}$. Next, we are going to refine it to obtain an example with $\partial_{C}^{+} M=\emptyset$, and so, ensuring that only the proper Busemann boundary plays a role.

Example 5.9 (Even when $\left(M, d^{+}\right)$is complete -and (5.3) holds- $\hat{j}$ may be neither surjective nor continuous.) Consider $V=\mathbb{R} \times \mathbb{R}$ and three metrics $g_{c l}, g, g_{\text {op }}$ such that:

- $g_{c l}$ and $g_{o p}$ are metrics as in 4.2 with $\omega \equiv 0, h=d x^{2}$ and $\alpha(t)=\frac{1}{e^{-t}+1}$ (clearly, the inequality (5.3) holds) and,

- $g$ is a metric as in (4.1) with $\omega_{t} \equiv 0$.

In order to ensure condition (4.3), we assume that $h_{t}$ satisfies (recall Proposition 4.5):

$$
\alpha(t) d x^{2} \leqslant h_{t} \leqslant d x^{2} .
$$

Consider the curve $\gamma(t)=(t, t), t \in \mathbb{R}$, which is lightlike for $g_{c l}$ and defines the TIP $P_{c l}=I_{c l}^{-}(\gamma)$. Such a TIP is characterized by the Busemann function $b_{c, c l}^{+}(\cdot)=\lim _{t \rightarrow \infty}(t-d(\cdot, c(t)))<\infty$, with $c(t)=t$, and $d$ is the Euclidean distance in $\mathbb{R}$. As $g_{c l} \prec g_{o p}, \gamma$ is also causal for $g_{o p}$, and so, it also defines a TIP for the metric $g_{o p}, P_{o p}=I_{o p}^{-}(\gamma)$. According to expressions (3.3) and (5.1) with $\tilde{\alpha}=\alpha$, this TIP is characterized by the following function:

$$
\begin{aligned}
b_{c, \text { op }}^{+}(\cdot) & =\lim _{t \rightarrow \infty}\left(\int_{0}^{t} \frac{1}{\alpha(s)} d s-d(\cdot, t)\right) \\
& =\lim _{t \rightarrow \infty}\left(\int_{0}^{t}\left(\frac{1}{\alpha(s)}-1\right) d s+(t-d(\cdot, t))\right) \\
& =\int_{0}^{\infty} e^{-s} d s+\lim _{t \rightarrow \infty}(t-d(\cdot, t))=1+b_{c, c l}^{+}(\cdot)<\infty,
\end{aligned}
$$

i.e., $P_{o p} \neq V$. Taking into account that $g_{o p}$ is (conformal to) a standard static spacetime, the result [7, Remark 4.17(1)] ensures the existence of some reparametrization

\footnotetext{
${ }^{4}$ That example is written in a different context, and does not satisfy 5.3 . However, it is trivial to modify it in order to ensure that 5.3 is fulfilled. The example also has other remarkable properties, as it shows explicitly a "lightlike strain" in $\hat{\partial} V$ which forbides the existence of a isomorphism (including the causal level) from $\hat{\partial} V$ to the timelike c-boundary $\hat{\partial}_{c l} V$.
} 
$\bar{c}$ of $c(t)=t$ such that $b_{\bar{c}, o p}^{+}=b_{c, o p}^{+}$and $\alpha(t)|\dot{\bar{c}}(t)|=1$, i.e. the curve $\bar{\rho}(t)=(t, \bar{c}(t))$, $t \in \mathbb{R}$ is lightlike for $g_{o p}$ (recall Remark 4.4). Now, put $l=\bar{c}^{-1}$ and reparameterize $\bar{\rho}$ as $\rho(s)=(l(s), s), s \in \mathbb{R}$. From $b_{\bar{c}, o p}^{+}=b_{c, o p}^{+}$, it is deduced that $\lim _{s \rightarrow \infty}(l(s)-s)=0$. This limit plus $l(s)=\alpha(l(s))<1$ implies $l(s)>s$ for all $s$ (see Figure 4).

Let us assume now some additional hypotheses for $h_{t}$ : suppose that $h_{t} \equiv \alpha(t) d x^{2}$ (resp. $h_{t} \equiv d x^{2}$ ) at each point $(t, x)$ such that $t \geq l(x)$ (resp. $\left.t \leq x\right)$, i.e., suppose that the metric $g$ coincides with $g_{o p}$ above the curve $\rho$ (resp. coincides with $g_{c l}$ below the curve $\gamma$ ). Now, for each $k \in(0, l(0))$, define a function $l_{k}: \mathbb{R} \rightarrow \mathbb{R}$ as the maximal solution of the following initial value problem:

$$
\left\{\begin{array}{l}
i_{k}(t)=h_{l_{k}(t)}\left(\frac{\partial}{\partial x}, \frac{\partial}{\partial x}\right) \\
l_{k}(0)=k
\end{array}\right.
$$

The curve $\gamma_{k}: \mathbb{R} \rightarrow \mathbb{R}^{2}$ defined by $\gamma_{k}(t)=\left(l_{k}(t), t\right)$ satisfies:

- It is lightlike for $g$ (apply Remark 4.4 with $F_{t}^{+}=h_{t}$ ), thus it determines a TIP $P^{k} \in \hat{\partial} V$.

- $l_{k}(t)$ satisfies that $t<l_{k}(t)<l(t)$. Otherwise, as $0<k=l_{k}(0)<l(0)$, the curve $\gamma_{k}$ should intersect either $\rho$ or $\gamma$ at some point $t_{0}$ (see Figure 4). But if, for example, $l_{k}\left(t_{0}\right)=l\left(t_{0}\right)$, the uniqueness of the solution to the first equality in (5.6) with initial data at $t_{0}$ would imply $l_{k} \equiv l$ (recall that $h_{t} \equiv \alpha(t) d x^{2}$ at $\gamma_{k}\left(t_{0}\right)=\rho\left(t_{0}\right)$, and the definitions of $l_{k}$ and $\left.l\right)$. Therefore, $t<l_{k}(t)<l(t)$, and so, $P_{c l} \subset P^{k} \subset P_{o p}=I_{o p}^{-}\left(P_{c l}\right)$. In particular, we deduce that $P_{c l} \subset P^{k}$ and $I_{o p}^{-}\left(P^{k}\right)=P_{o p}$.

- By construction, if $k_{1}, k_{2} \in(0, l(0))$ satisfy $k_{1}<k_{2}$, then $P^{k_{1}} \subsetneq P^{k_{2}}$. In fact, the uniqueness of the solution for the initial value problem ensures that $l_{k_{1}}(t)<l_{k_{2}}(t)$ for all $t$, and so, the inclusion $\subset$ is trivially obtained. Moreover, this inclusion is strict, since any past-directed timelike curve from a point in $\gamma_{k_{1}}$ must remain below this curve (the past cone at any point of $\gamma_{k_{1}}$ points out downward the image of the curve).

Hence, for every $k \in(0, l(0))$ there exists a TIP $P^{k}$ for $g$ such that $P_{c l} \subset P^{k} \subset$ $I_{o p}^{-}\left(P^{k}\right)=I_{o p}^{-}\left(P_{c l}\right)$. In particular, the map $\hat{j}$ defined in Theorem 5.5 cannot be surjective since, in that case, and according to Remark 5.8, each $P^{k}$ should be the image of $P_{c l}$, which is impossible. Moreover, $\hat{j}$ is not continuous; for instance, the sequence $\left\{I_{c l}^{-}\left(\gamma_{k}(n)\right)\right\}_{n}$ converges to $P_{c l}$ in $\left(V, g_{c l}\right)$ but $\left\{\hat{j}\left(I_{c l}^{-}\left(\gamma_{k}(n)\right)\right)\right\}_{n}=\left\{I^{-}\left(\gamma_{k}(n)\right)\right\}_{n}$ converges to $P^{k} \neq \hat{j}\left(P_{c l}\right)=I^{-}\left(P_{c l}\right)$ in $(V, g)$.

\subsection{Strains and topological information}

A reason for the lack of continuity of $\hat{j}$ is the existence of different TIPs for the metric $g$ sharing the same past when computed with the metric $g_{\text {op }}$ (recall Remark 5.8). In principle, this also indicates the existence of alternative choices for $\hat{j}$. In order to overcome this problem, we are going to identify all these TIPs by passing to 




Figure 4: Qualitative behavior of functions $\rho, \gamma_{n}$ and $\gamma$ in Example 5.9.

the corresponding quotient space. To this aim, first we need the following technical lemma.

Lemma 5.10 Suppose that (5.3) holds and consider an IP $P \in \hat{V}$ for the metric $g$ and $P_{o p}:=I_{o p}^{-}(P)$. Then, there exists an unique $P_{c l} \in \hat{V}_{c l}$ satisfying:

$$
P_{c l} \subset P \text { and } I_{o p}^{-}\left(P_{c l}\right)=P_{o p}
$$

Proof. Suppose that $P=I^{-}(\gamma)$ with $\gamma:[a, \Omega) \rightarrow V, \gamma(s)=(s, c(s))$ a futuredirected timelike curve for $g$. Observe that, assumed the existence of such a TIP $P_{c l}$, the uniqueness follows from Proposition 5.3 (2). For the existence, we will consider two cases for clarity.

In one hand, assume that $\Omega<\infty$. In this case, $c(s) \rightarrow x^{+} \in \partial_{C}^{+} M$ as $s \rightarrow \Omega$ $\left(\gamma\right.$ is causal in $\left.\left(V, g_{o p}\right)\right)$. Consider a timelike curve for $g_{c l}, \gamma_{c l}:[a, \Omega) \rightarrow V$, of the form $\gamma_{c l}(s)=\left(t_{c l}(s), c(s)\right)$ where $t_{c l}(s)<s$ and $t_{c l}(s) \rightarrow \Omega$. This curve can be constructed by taking

$$
\dot{t}_{c l}(s)>\frac{1}{\alpha_{0}}>\frac{F_{s}^{+}(\dot{c}(s))}{\alpha_{0}} \geq F^{+}(\dot{c})
$$

where $\alpha_{0}=\left.\min \right|_{[a, \Omega]}(\alpha(s))$ (recall that $\gamma$ is timelike for $g$, i.e. $F_{s}^{+}(\dot{c})<1$, and (4.6)). From the construction, $P_{c l}=I_{c l}^{-}\left(\gamma_{c l}\right) \subset P$ and, as both curves end at the same pair $\left(\Omega, x^{+}\right)$, necessarily $I_{o p}^{-}\left(P_{o p}\right)=I_{o p}^{-}(P)$ (recall $\left.(3.9)\right)$.

In the other hand, suppose that $\Omega=\infty$. First, consider the curve $\gamma_{c l}(s)=$ $\left(t_{c l}(s), c(s)\right)$, with $s \in[a, \infty)$ and where the component $t_{c l}(s)$ is determined by the Finsler metrics in (4.4) and 4.5) as:

$$
\frac{d t_{c l}}{d s}=\frac{F^{+}(\dot{c})}{F_{s}^{+}(\dot{c})}, \quad t_{c l}(a)=-k_{c l}:=-\int_{a}^{\infty}\left(\frac{F^{+}(\dot{c})}{F_{s}^{+}(\dot{c})}-1\right) .
$$


Note that $k_{c l}$ is finite. In fact, from 4.6 it is $1 \leqslant F^{+}(\dot{c}) / F_{s}^{+}(\dot{c}) \leqslant 1 / \alpha(s)$, which joined to $(5.3)$ imply

$$
(0 \leqslant) k_{c l}=\int_{a}^{\infty}\left(\frac{F^{+}(\dot{c})}{F_{s}^{+}(\dot{c})}-1\right) \leqslant \int_{a}^{\infty}\left(\frac{1}{\alpha(s)}-1\right)<\infty .
$$

Moreover, as the curve $\gamma(s)$ is future-directed timelike for $g$, i.e. $F_{s}^{+}(\dot{c})<1$, so is the curve $\gamma_{c l}$ for $g_{c l}$, since

$$
F^{+}(\dot{c})<\frac{F^{+}(\dot{c})}{F_{s}^{+}(\dot{c})}=\frac{d t_{c l}}{d s} .
$$

Similarly, one can consider the curve $\gamma_{o p}(s)=\left(t_{o p}(s), c(s)\right)$, where the component $t_{o p}(s)$ is determined by:

$$
\frac{d t_{o p}}{d s}=\frac{\alpha(s) F^{+}(\dot{c})}{F_{s}^{+}(\dot{c})}, \quad t_{o p}(a)=k_{o p}:=\int_{a}^{\infty}\left(1-\frac{\alpha(s) F^{+}(\dot{c})}{F_{s}^{+}(\dot{c})}\right) .
$$

Again, $k_{o p}$ is finite since, from 4.6 , it is $\alpha(s) \leqslant \alpha(s) F^{+}(\dot{c}) / F_{s}^{+}(\dot{c}) \leqslant 1$, which joined to 5.3 implies

$$
\begin{aligned}
(0 \leqslant) k_{o p} & =\int_{a}^{\infty}\left(1-\frac{\alpha(s) F^{+}(\dot{c})}{F_{s}^{+}(\dot{c})}\right) \leqslant \int_{a}^{\infty}(1-\alpha(s))= \\
& =\int_{a}^{\infty} \alpha(s)\left(\frac{1}{\alpha(s)}-1\right) \leqslant \int_{a}^{\infty}\left(\frac{1}{\alpha(s)}-1\right)<\infty .
\end{aligned}
$$

With this definition, the curve $\gamma_{o p}$ is future-directed timelike for $g_{o p}$, since

$$
\alpha(s) F^{+}(\dot{c})<\alpha(s) \frac{F^{+}(\dot{c})}{F_{s}^{+}(\dot{c})}=\frac{d t_{o p}}{d s} .
$$

Note also that $\lim _{s \rightarrow \infty}\left(t_{o p}(s)-t_{c l}(s)\right)=0$. In fact:

$$
\begin{aligned}
\lim _{s \rightarrow \infty}\left(t_{o p}(s)-t_{c l}(s)\right) & =\lim _{s \rightarrow \infty}\left(t_{o p}(s)-s+s-t_{c l}(s)\right) \\
& =\lim _{s \rightarrow \infty}\left(\int_{a}^{s} \frac{d t_{o p}}{d s^{\prime}} d s^{\prime}+k_{o p}-s+s-\int_{a}^{s} \frac{d t_{c l}}{d s^{\prime}} d s^{\prime}+k_{c l}\right) \\
& =\lim _{s \rightarrow \infty}\left(\int_{a}^{s}\left(\frac{d t_{o p}}{d s^{\prime}}-1\right) d s^{\prime}+k_{o p}-\int_{a}^{s}\left(\frac{d c_{c l}}{d s^{\prime}}-1\right) d s^{\prime}+k_{c l}\right) \\
& =\int_{a}^{\infty}\left(\frac{d t_{o p}}{d s}-1\right) d s-\int_{a}^{\infty}\left(\frac{d t_{c l}}{d s}-1\right) d s+k_{o p}+k_{c l} \\
& =\int_{a}^{\infty}\left(\frac{\alpha(s) F^{+}(\dot{c})}{F_{s}^{+}(\dot{c})}-1\right) d s-\int_{a}^{\infty}\left(\frac{F^{+}(\dot{c})}{F_{s}^{+}(\dot{c})}-1\right) d s+k_{o p}+k_{c l} \\
& =0 .
\end{aligned}
$$

Hence, by construction it is clear that $t_{c l}(s) \leqslant s \leqslant t_{o p}(s)$ for all $s \in[a, \infty)$. Therefore, if one defines the following TIPs for $g_{c l}$ and $g_{o p}$, resp.,

$$
P_{c l}:=I_{c l}^{-}\left(\gamma_{c l}\right) \quad P_{o p}^{*}:=I_{o p}^{-}\left(\gamma_{o p}\right),
$$

one automatically has these inclusions:

$$
P_{c l} \subset P \subset P_{o p}^{*}, \quad \text { and thus } \quad I_{o p}^{-}\left(P_{c l}\right) \subset P_{o p}\left(=I_{o p}^{-}(P)\right) \subset I_{o p}^{-}\left(P_{o p}^{*}\right)=P_{o p}^{*} .
$$


So, in order to obtain the required equality $I_{o p}^{-}\left(P_{c l}\right)=P_{o p}$, it is sufficient to prove

$$
I_{o p}^{-}\left(P_{c l}\right)=P_{o p}^{*}
$$

Define the curves $c_{c l}(s):=c\left(t_{c l}^{-1}(s)\right), c_{o p}(s):=c\left(t_{o p}^{-1}(s)\right)$. Then,

$$
\begin{array}{cc}
P_{c l}=I_{c l}^{-}\left(\tilde{\gamma}_{c l}\right), & \text { where } \tilde{\gamma}_{c l}(s):=\left(s, c_{c l}(s)\right) \\
P_{o p}^{*}=I_{o p}^{-}\left(\tilde{\gamma}_{o p}\right), & \text { where } \tilde{\gamma}_{o p}(s):=\left(s, c_{o p}(s)\right)
\end{array}
$$

From the study developed in Section 3.1, the TIPs $I_{o p}^{-}\left(P_{c l}\right), P_{o p}^{*}$ can be rewritten as follows (recall $(3.3)$ and $(3.4)$ ):

$$
I_{o p}^{-}\left(P_{c l}\right)=P_{o p}\left(b_{c_{c l}, o p}^{+}\right), \quad P_{o p}^{*}=P_{o p}\left(b_{c_{o p}, o p}^{+}\right)
$$

So, 5.8 will follow if we prove that $b_{c_{o p}, o p}^{+}=b_{c_{c l}, o p}^{+}$. To this aim, first note that

$$
\begin{aligned}
& b_{c_{c l}, o p}^{+}(\cdot)=\lim _{s \rightarrow \infty}\left(\int_{0}^{s} \frac{d s^{\prime}}{\alpha\left(s^{\prime}\right)}-d^{+}\left(\cdot, c_{c l}(s)\right)\right)=\lim _{s \rightarrow \infty}\left(\int_{0}^{t_{c l}(s)} \frac{d s^{\prime}}{\alpha\left(s^{\prime}\right)}-d^{+}(\cdot, c(s))\right) \\
& b_{c_{o p}, o p}^{+}(\cdot)=\lim _{s \rightarrow \infty}\left(\int_{0}^{s} \frac{d s^{\prime}}{\alpha\left(s^{\prime}\right)}-d^{+}\left(\cdot, c_{o p}(s)\right)\right)=\lim _{s \rightarrow \infty}\left(\int_{0}^{t_{o p}(s)} \frac{d s^{\prime}}{\alpha\left(s^{\prime}\right)}-d^{+}(\cdot, c(s))\right) .
\end{aligned}
$$

Thus, from (5.3) and (5.7), one deduces

$$
\begin{aligned}
b_{c_{o p}, o p}^{+}(\cdot)-b_{c_{c l}, o p}^{+}(\cdot) & =\lim _{s \rightarrow \infty}\left(\int_{0}^{t_{o p}(s)} \frac{d s^{\prime}}{\alpha\left(s^{\prime}\right)}-\int_{0}^{t_{c l}(s)} \frac{d s^{\prime}}{\alpha\left(s^{\prime}\right)}\right) \\
& =\lim _{s \rightarrow \infty}\left(\int_{t_{c l}(s)}^{t_{o p}(s)} \frac{d s^{\prime}}{\alpha\left(s^{\prime}\right)}\right) \\
& =\lim _{s \rightarrow \infty}\left(\int_{t_{c l}(s)}^{t_{o p}(s)}\left(\frac{1}{\alpha\left(s^{\prime}\right)}-1\right) d s^{\prime}+\int_{t_{c l}(s)}^{t_{o p}(s)} d s^{\prime}\right) \\
& =0 .
\end{aligned}
$$

We are now in conditions to introduce the following definition.

Definition 5.11 Two TIPs $P^{1}, P^{2} \in \hat{\partial} V$ are st-related, $P^{1} \sim_{s t} P^{2}$, if there exists a TIP $P_{c l}$ for $g_{c l}$ such that $P_{c l} \subset P^{1} \cap P^{2}$ and $I_{o p}^{-}\left(P^{1}\right)=I_{o p}^{-}\left(P_{c l}\right)=I_{o p}^{-}\left(P^{2}\right)$.

When (5.3) holds, this relation is of equivalenc $\AA^{5}$ and it defines the quotient space $\hat{V} / \sim_{s t}$. The (non-trivial) classes of equivalence in $\hat{V} / \sim_{s t}$ will be called (future) strains.

We will denote by $\hat{\Pi}$ the natural projection onto the quotient, $\hat{\Pi}: \hat{V} \rightarrow \hat{V} / \sim_{s t}$.

Remark 5.12 The space $\hat{V} / \sim_{s t}$ is endowed with the quotient topology from $\hat{V}$. Here, we are going to justify that this quotient topology can be also given as well in terms of a limit operator. The argument below is general.

Let $L$ be a limit operator on a set $X$, i.e., a map $L: S(X) \rightarrow \mathcal{P}(X)$ (where $S(X)$ is the set of all sequences in $X$ and $\mathcal{P}(X)$ is the set of parts of $X$ ) satisfying that, if $\tilde{\sigma}$ is a subsequence of some $\sigma \in S(X)$, then $L(\sigma) \subset L(\tilde{\sigma})$. Endow $X$ with the

\footnotetext{
${ }^{5}$ Symmetry and transitivity are straightforward, while reflexivity is a consequence of Lemma 5.10
} 
associated topology $\tau^{L}$, defined by the following statement: $C \subset X$ is closed if, and only if, $L(\sigma) \subset C$ for all sequence $\sigma$ in $C$. Define in $X$ a relation of equivalence $\sim$ and denote by $\pi: X \rightarrow X / \sim$ the natural projection. The space $X / \sim$ is endowed with the quotient topology induced from $\tau^{L}$ and $\sim$. Alternatively, note that $L$ and $\sim$ allow to define a limit operator $L_{\sim}$ in the quotient $X / \sim$ just by putting 6

$$
[x] \in L_{\sim}\left(\left\{\left[x_{n}\right]\right\}\right) \Longleftrightarrow \exists x^{\prime} \in \pi^{-1}([x]), x_{n}^{\prime} \in \pi^{-1}\left(\left[x_{n}\right]\right) \forall n \in \mathbb{N}: x^{\prime} \in L\left(\left\{x_{n}^{\prime}\right\}\right) .
$$

where $[x],\left[x_{n}\right] \in X / \sim$. The equivalence between both topologies on $X / \sim$, the topology $\tau^{L}$ associated to $L_{\sim}$ and the quotient topology $\tau^{L} / \sim$ induced from $\tau^{L}$ and $\sim$, comes from the following equivalences:

$$
\begin{aligned}
C \subset X / \sim \text { is closed for } \tau / \sim & \Longleftrightarrow \pi^{-1}(C) \text { is closed in } X \\
& \Longleftrightarrow L(\sigma) \subset \pi^{-1}(C) \text { for all sequence } \sigma \subset \pi^{-1}(C) \\
& \Longleftrightarrow L_{\sim}(\pi(\sigma)) \subset C \text { for all sequence } \pi(\sigma) \subset C .
\end{aligned}
$$

Next, we are going to determine when the composition $\hat{\mathcal{J}}:=\hat{\Pi} \circ \hat{j}$ is bijective and continuous. But, first, we need some previous lemmas.

Lemma 5.13 Assume that 5.3) holds, $d_{Q}^{+}$is a generalized distance and $M_{C}^{+}$is locally compact. Then, $P_{c l} \in \hat{L}\left(\left\{P_{c l}^{n}\right\}\right)$ if and only if $P_{o p} \in \hat{L}\left(\left\{P_{o p}^{n}\right\}\right)$, where $P_{o p}=$ $I_{o p}^{-}\left(P_{c l}\right)$ and $P_{o p}^{n}=I_{o p}^{-}\left(P_{c l}^{n}\right)$.

Proof. Consider $\gamma_{n}:\left[a_{n}, \Omega_{n}\right) \rightarrow V, \gamma:[a, \Omega) \rightarrow V$ such that $\gamma_{n}=\left(t, c_{n}(t)\right)$, $\gamma(t)=(t, c(t))$ and $P_{c l}^{n}=P_{c l}\left(b_{c_{n}, c l}^{+}\right)=I_{c l}^{-}\left(\gamma_{n}\right), P_{c l}=P_{c l}\left(b_{c, c l}^{+}\right)=I_{c l}^{-}(\gamma)$. Recalling (3.3), 3.4, we deduce that $I_{o p}^{-}\left(\gamma_{n}\right)=P_{o p}\left(b_{c_{n}, o p}^{+}\right)$and $I_{o p}^{-}(\gamma)=P_{o p}\left(b_{c, o p}^{+}\right)$. In particular, our statement is equivalent to the following one (recall Section 3.3):

$$
b_{c, c l}^{+} \in \hat{L}\left(\left\{b_{c_{n}, c l}^{+}\right\}\right) \text {if and only if } b_{c, o p}^{+} \in \hat{L}\left(\left\{b_{c_{n}, o p}^{+}\right\}\right) .
$$

We will focus on the implication to the left, as the other one is similar but simpler. So, assume that $b_{c, \text { op }}^{+} \in \hat{L}\left(\left\{b_{c_{n}, o p}^{+}\right\}\right)$. From Proposition 5.3. we have that $b_{c_{n}, \text { op }}^{+}=$ $b_{c_{n}, c l}^{+}+K_{\Omega_{n}}$ and $b_{c, o p}^{+}=b_{c, c l}^{+}+K_{\Omega}$. Then, from our assumption, we deduce that $b_{c, c l}^{+} \in \hat{L}\left(\left\{b_{c_{n}, c l}^{+}+\left(K_{\Omega_{n}}-K_{\Omega}\right)\right\}_{n}\right)$. The result follows if we prove that $K_{\Omega_{n}} \rightarrow K_{\Omega}$ or, equivalently, that $\Omega_{n} \rightarrow \Omega$ (recall (5.3)).

On one hand, suppose that $\Omega<\infty$ and define:

$$
\Omega_{o p}^{n}=\int_{0}^{\Omega_{n}} \frac{d s}{\alpha(s)}, \quad \Omega_{o p}=\int_{0}^{\Omega} \frac{d s}{\alpha(s)}
$$

With these definitions, we obtain that $b_{c, o p}^{+}=d_{\left(\Omega_{o p}, x^{+}\right)}^{+}$for some $x^{+} \in M_{C}^{+}$(recall (3.7) and (3.8)). As $M_{C}^{+}$is locally compact, $d_{Q}^{+}$is a generalized distance and

\footnotetext{
${ }^{6}$ It is worth pointing out that this is not the unique possible choice for $L_{\sim}$ (a drawback of $L_{\sim}$ is that, even if $L$ is of the first order, $L \sim$ may not). However, other natural alternatives would yield the same topology.
} 
$d_{\left(\Omega_{o p}, x^{+}\right)}^{+}=b_{c, o p}^{+} \in \hat{L}\left(\left\{b_{c_{n}, o p}^{+}\right\}\right)$, the naturally adapted version of [7, Proposition 5.24] ensures that $\left\{\Omega_{o p}^{n}\right\} \rightarrow \Omega_{o p}$, and so, that $\Omega_{n} \rightarrow \Omega$.

On the other hand, if $\Omega=\infty$, as $\gamma(t) \in P_{o p} \subset \operatorname{LI}\left(\left\{P_{o p}^{n}\right\}\right)$, for every $t \in[a, \infty)$ there exists $n_{0}$ such that $\gamma(t)=(t, c(t)) \in P_{o p}^{n}$ for $n \geq n_{0}$. Therefore, $\gamma(t) \ll_{o p} \gamma_{n}\left(t^{\prime}\right)$ for some $t^{\prime} \in\left[a_{n}, \Omega_{n}\right)$, and, from Prop. 3.1.

$$
\int_{t}^{t^{\prime}} \frac{d s}{\alpha(s)}>d^{+}\left(c(t), c_{n}\left(t^{\prime}\right)\right) \geq 0
$$

which implies that $\Omega_{n}\left(>t^{\prime}\right) \geq t$. Taking $t \nearrow \infty$, one deduces that $\Omega_{n} \rightarrow \Omega=\infty$.

Lemma 5.14 Under the hypotheses of Lemma 5.13, if $P_{c l} \in \hat{L}\left(\left\{P_{c l}^{n}\right\}\right)$ then any sequence $\left\{P^{n}\right\}_{n}$, with $P^{n} \in \hat{\Pi}^{-1}\left(\hat{\mathcal{J}}\left(P_{c l}^{n}\right)\right)$, contains some subsequence $\left\{P^{n_{k}}\right\}_{k}$ such that $P \in \hat{L}\left(\left\{P^{n_{k}}\right\}\right)$ for some $P \in \hat{\Pi}^{-1}\left(\hat{\mathcal{J}}\left(P_{c l}\right)\right)$.

Before the proof of this lemma, we have to introduce the following general result for c-completions (recall [3, Corollary 5.12]). For the convenience of the reader, a detailed proof of this result is included here.

Lemma 5.15 Take $\left\{P^{n}\right\} \subset \hat{V}$ and $P^{\prime} \in \hat{V}$. If $P^{\prime} \subset \operatorname{LI}\left(\left\{P^{n}\right\}\right)$ then there exist $P \supset P^{\prime}$ and a subsequence $\left\{P^{n_{k}}\right\}_{k} \subset\left\{P^{n}\right\}$ such that $P \in \hat{L}\left(\left\{P^{n_{k}}\right\}_{k}\right)$.

Proof. As $P^{\prime} \subset \operatorname{LI}\left(\left\{P^{n}\right\}\right) \subset \operatorname{LS}\left(\left\{P^{n}\right\}\right)$, Zorn's lemma ensures the existence of a maximal IP $P$ such that $P^{\prime} \subset P \subset \operatorname{LS}\left(\left\{P^{n}\right\}\right)$. Let $\left\{x_{m}\right\} \subset V$ be a chronological chain (i.e. $x_{m} \ll x_{m+1}$ for all $m$ ) generating $P$ (i.e. $P=\cup_{m} I^{-}\left(x_{m}\right)$ ). As $P \subset$ $\operatorname{LS}\left(\left\{P^{n}\right\}\right)$, for every $m$ there exists $\left\{n_{k}^{m}\right\}_{k} \subset \mathbb{N}$ such that $x_{m} \in P^{n_{k}^{m}}$ for all $k$. As $x_{m} \ll x_{m+1}$, the desired subsequence is $\left\{P^{n_{k}}\right\}_{k}$ with $n_{k}:=n_{k}^{k}$. In fact, by construction $P$ satisfies: (i) $P \subset \operatorname{LI}\left(\left\{P^{n_{k}}\right\}\right.$ ), (ii) $P$ is maximal in $\operatorname{LS}\left(\left\{P^{n_{k}}\right\}\right)$ (as $P$ is maximal in $\left.\operatorname{LS}\left(\left\{P^{n}\right\}\right)\right)$, and the first assertion follows.

Proof of Lemma 5.14. As $P_{c l} \in \hat{L}\left(\left\{P_{c l}^{n}\right\}\right)$, necessarily $P_{c l} \subset \operatorname{LI}\left(\left\{P_{c l}^{n}\right\}\right)$, and thus, $P_{c l} \subset \operatorname{LI}\left(\left\{I^{-}\left(P_{c l}^{n}\right)\right\}\right)$. As the inferior limit is a past set for $g$, necessarily

$$
\hat{j}\left(P_{c l}\right)=I^{-}\left(P_{c l}\right) \subset \operatorname{LI}\left(\left\{I^{-}\left(P_{c l}^{n}\right)\right\}\right)=\operatorname{LI}\left(\left\{\hat{j}\left(P_{c l}^{n}\right)\right\}\right) .
$$

Moreover, since any element $P^{n} \in \hat{\Pi}^{-1}\left(\hat{\mathcal{J}}\left(P_{c l}^{n}\right)\right)$ satisfies $P_{c l}^{n} \subset P^{n}$ (Defn. 5.11, necessarily $I^{-}\left(P_{c l}^{n}\right) \subset I^{-}\left(P^{n}\right)=P^{n}$. Taking into account 5.11, it is

$$
\hat{j}\left(P_{c l}\right) \subset \operatorname{LI}\left(\left\{I^{-}\left(P_{c l}^{n}\right)\right\}\right) \subset \operatorname{LI}\left(\left\{P^{n}\right\}\right) \subset \operatorname{LS}\left(\left\{P^{n}\right\}\right),
$$

and $\hat{j}\left(P_{c l}\right)$ is an IP for $g$. Then, from Lemma 5.15 there exist an IP $P$ for $g$ and some subsequence $\left\{P^{n_{k}}\right\}_{k} \subset\left\{P^{n}\right\}$ such that:

$$
P \in \hat{L}\left(\left\{P^{n_{k}}\right\}\right) .
$$

In order to check that $P \in \hat{\Pi}^{-1}\left(\hat{\mathcal{J}}\left(P_{c l}\right)\right)$, note that from $g \prec_{0} g_{o p}$ and the inclusion $\hat{j}\left(P_{c l}\right) \subset P$,

$$
I_{o p}^{-}\left(P_{c l}\right)=I_{o p}^{-}\left(I^{-}\left(P_{c l}\right)\right) \subset I_{o p}^{-}(P) .
$$


Taking into account that $P^{n_{k}} \in \hat{\Pi}^{-1}\left(\hat{\mathcal{J}}\left(P_{c l}^{n_{k}}\right)\right)$ and 5.12 , it is straightforward that

$$
\left.I_{o p}^{-}(P) \subset \operatorname{LI}\left(\left\{I_{o p}^{-}\left(P^{n_{k}}\right)\right\}\right)\right)=\operatorname{LI}\left(\left\{I_{o p}^{-}\left(P_{c l}^{n_{k}}\right)\right\}\right) .
$$

Moreover, as $P_{c l} \in \hat{L}\left(\left\{P_{c l}^{n}\right\}\right)$, from Lemma 5.13 the subsequence satisfies $I_{o p}^{-}\left(P_{c l}\right) \in$ $\hat{L}\left(\left\{I_{o p}^{-}\left(P_{c l}^{n_{k}}\right)\right\}\right)$. Hence, $I_{o p}^{-}\left(P_{c l}\right)$ is maximal in $L S\left(\left\{I_{o p}^{-}\left(P_{c l}^{n_{k}}\right)\right\}\right)$ (recall 2.3p), which joined to $(5.13)$ and 5.14 implies

$$
I_{o p}^{-}\left(P_{c l}\right)=I_{o p}^{-}(P) .
$$

From this equality and the fact that $P_{c l} \subset I^{-}\left(P_{c l}\right) \subset P$, we deduce that $P \in$ $\hat{\Pi}^{-1}\left(\hat{\mathcal{J}}\left(P_{c l}\right)\right)$, as required.

Theorem 5.16 Let $V=(\mathbb{R} \times M, g)$ be a spacetime as in (4.1) such that $g_{c l} \prec_{0}$ $g \prec_{0} g_{\text {op }}$. If $(5.3)$ holds, $d_{Q}^{+}$is a generalized distance and $\bar{M}_{C}^{+}$is locally compact then the map

$$
\hat{\mathcal{J}}=\hat{\Pi} \circ \hat{j}: \hat{V}_{c l} \rightarrow \hat{V} / \sim_{s t}
$$

is bijective and continuous. Moreover, if, in addition, $\hat{V} / \sim_{\text {st }}$ is Hausdorff, then $\hat{\mathcal{J}}$ is an homeomorphism.

Proof. For the injectivity of $\hat{\mathcal{J}}$, assume that $P_{c l}^{1} \neq P_{c l}^{2}$. From Proposition 5.3 (2), $I_{o p}^{-}\left(\hat{j}\left(P_{c l}^{1}\right)\right)=I_{o p}^{-}\left(P_{c l}^{1}\right) \neq I_{o p}^{-}\left(P_{c l}^{2}\right)=I_{o p}^{-}\left(\hat{j}\left(P_{c l}^{2}\right)\right)$. So, $\hat{j}\left(P_{c l}^{1}\right) \psi_{s t} \hat{j}\left(P_{c l}^{2}\right)$ and then $\hat{\mathcal{J}}\left(P_{c l}^{1}\right) \neq \hat{\mathcal{J}}\left(P_{c l}^{2}\right)$.

For the continuity $]^{7}$ of $\hat{\mathcal{J}}$, consider a sequence $\left\{P_{c l}^{n}\right\} \subset \hat{V}_{c l}$ and $P_{c l} \in \hat{V}_{c l}$ such that $P_{c l} \in \hat{L}\left(\left\{P_{c l}^{n}\right\}\right)$. By contradiction, suppose that $\left\{\hat{\mathcal{J}}\left(P_{c l}^{n}\right)\right\}$ does not converge to $\hat{\mathcal{J}}\left(P_{c l}\right)$. Then, there exists an open neighborhood $U$ of $\hat{\mathcal{J}}\left(P_{c l}\right)$ such that, up to a subsequence, it does not contain $\left\{\hat{\mathcal{J}}\left(P_{c l}^{n}\right)\right\}$ for any $n$; i.e., $\hat{\Pi}^{-1}(U)$ is an open set of $\hat{V}$ containing all the elements in $\hat{\Pi}^{-1}\left(\hat{\mathcal{J}}\left(P_{c l}\right)\right)$, but not containing any element of $\hat{\Pi}^{-1}\left(\hat{\mathcal{J}}\left(P_{c l}^{n}\right)\right)$. This is in contradiction to the fact that, from Lemma 5.14, there exists some subsequence $\left\{P^{n_{k}}\right\}_{k}, P^{n_{k}} \in \hat{\Pi}^{-1}\left(\hat{\mathcal{J}}\left(P_{c l}^{n_{k}}\right)\right)$ and $P \in \hat{\Pi}^{-1}\left(\hat{\mathcal{J}}\left(P_{c l}\right)\right)$ such that $P \in \hat{L}\left(\left\{P^{n_{k}}\right\}\right)$.

For the surjectivity of $\hat{\mathcal{J}}$, consider an arbitrary element $\hat{\Pi}(P) \in \hat{V} / \sim_{s t}$, with $P$ IP of $(V, g)$. Lemma 5.10 ensures the existence of $P_{c l} \in \hat{V}_{c l}$ such that

$$
P_{c l} \subset P \text { and } I_{o p}^{-}\left(P_{c l}\right)=I_{o p}^{-}(P) .
$$

In particular, we deduce that $\left(\hat{j}\left(P_{c l}\right)=\right) I^{-}\left(P_{c l}\right) \sim_{s t} P$, and so, that $\hat{\mathcal{J}}\left(P_{c l}\right)=$ $\hat{\Pi}\left(\hat{j}\left(P_{c l}\right)\right)=\hat{\Pi}(P)$, as required.

Finally, let us prove that $\hat{\mathcal{J}}^{-1}$ is continuous if $\hat{V} / \sim_{s t}$ is Hausdorff. Consider a sequence $\left\{\hat{\mathcal{J}}\left(P_{c l}^{n}\right)\right\}_{n} \subset \hat{V} / \sim_{s t}$ and $\hat{\mathcal{J}}\left(P_{c l}\right) \in \hat{V} / \sim_{s t}$ such that $\hat{\mathcal{J}}\left(P_{c l}\right) \in$ $\hat{L}_{\sim_{s t}}\left(\left\{\hat{\mathcal{J}}\left(P_{c l}^{n}\right)\right\}\right)$ (where $\hat{L}_{\sim_{s t}}$ is the quotient limit associated to $\hat{L}$ and $\sim_{s t}$, recall

\footnotetext{
7 Notice that the simple characterization of continuity by means of sequences (which is used typically in first countable spaces) holds also when the domain is a sequential space defined by a limit operator, see [6] Footnote 13].
} 
(5.9)). We have just to check that $P_{c l} \in \hat{L}\left(\left\{P_{c l}^{n}\right\}\right)$, and so, $\left\{P_{c l}^{n}\right\}$ converges to $P_{c l}$ with the future chronological topology (recall again footnote 7 ). Assume by contradiction that this is not the case. From the definition of $L_{\sim_{s t}}$, there exist a sequence $\left\{P^{n}\right\}$, with $P^{n} \in \hat{\Pi}^{-1}\left(\hat{\mathcal{J}}\left(\left\{P_{c l}^{n}\right\}\right)\right)$, and some $P \in \hat{\Pi}^{-1}\left(\hat{\mathcal{J}}\left(P_{c l}\right)\right)$ such that $P \in \hat{L}\left(\left\{P^{n}\right\}\right)$. In particular, from 2.3$)$ and the fact that $g \prec g_{o p}$, $P_{o p}:=I_{o p}^{-}(P) \subset L I\left(\left\{I_{o p}^{-}\left(P^{n}\right)\right\}\right)=L I\left(\left\{P_{o p}^{n}\right\}\right)$. Observe that $P_{o p} \notin \hat{L}\left(\left\{P_{o p}^{n}\right\}\right)$ as, otherwise, Lemma 5.13 ensures that $P_{c l} \in \hat{L}\left(\left\{P_{c l}^{n}\right\}\right)$, a contradiction (observe that, as $P \in \hat{\Pi}^{-1}\left(\hat{\mathcal{J}}\left(P_{c l}\right)\right)$ and $P^{n} \in \hat{\Pi}^{-1}\left(\hat{\mathcal{J}}\left(P_{c l}^{n}\right)\right)$, necessarily $P_{o p}=I_{o p}^{-}\left(P_{c l}\right)$ and $\left.P_{o p}^{n}=I_{o p}^{-}\left(P_{c l}^{n}\right)\right)$. So, Lemma 5.15 ensures the existence of a subsequence $\left\{P_{o p}^{n_{k}}\right\}_{k}$ of $\left\{P_{o p}^{n}\right\}$ and $P_{o p} \subsetneq P_{o p}^{\prime} \in \hat{V}_{o p}$ such that $P_{o p}^{\prime} \in \hat{L}\left(\left\{P_{o p}^{n_{k}}\right\}\right)$. Now, taking into account that $P_{o p}^{\prime}=I_{o p}^{-}\left(P_{c l}^{\prime}\right)$ for some $P_{c l}^{\prime} \in \hat{V}_{c l}$ (apply Lemma 5.10 with $g=g_{o p}$ ) and applying Lemma 5.13, we obtain that $P_{c l}^{\prime} \in \hat{L}\left(\left\{P_{c l}^{n_{k}}\right\}\right)$ with $P_{c l}^{\prime} \neq P_{c l}\left(\right.$ as $\left.P_{o p}^{\prime} \neq P_{o p}\right)$. Finally, from the injectivity and continuity of $\hat{\mathcal{J}}$,

$$
\hat{\mathcal{J}}\left(P_{c l}\right), \hat{\mathcal{J}}\left(P_{c l}^{\prime}\right) \in \hat{L}_{\sim_{s t}}\left(\left\{\hat{\mathcal{J}}\left(P_{c l}^{n_{k}}\right)\right\}\right) \text { with } \hat{\mathcal{J}}\left(P_{c l}\right) \neq \hat{\mathcal{J}}\left(P_{c l}^{\prime}\right),
$$

which contradicts the Hausdorff character of $\hat{V} / \sim_{s t}$.

Remark 5.17 Under general circumstances (for example, open subsets of manifolds) a continuous bijective map admits a continuous inverse. However, the isocausality between $V$ and $V_{c l}$ does not imply the Hausdorffness of $\hat{V}$ nor $\hat{V} / \sim_{s t}$ and, obviously, the latter is an obstruction for $\hat{\mathcal{J}}$ being an homeomorphism (recall that $\hat{V}_{c l}$ is Hausdorff). In order to understand why $\hat{V}$ may be non-Hausdorff, recall that the $\hat{L}$-convergence of a sequence of TIP's $\left\{P^{n}\right\}$ to some TIP $P \subset \liminf \left\{P^{n}\right\}$ depends on the maximality of $P$ into $\lim \sup \left\{P^{n}\right\}$. The hypotheses on $g_{c l}$ (formulated by using the Fermat metric for stationary spacetimes) prevent the existence of different maximal sets in $\lim \sup \left\{P^{n}\right\}$, but there is no reason to expect that such sets do not exist for $g$-namely, a close but non-stationary metric. Nevertheless, the last statement of Theorem 5.16 shows that non-Hausdorffness is the unique obstruction for the continuity of $\mathcal{J}^{-1}$.

\subsection{Past c-boundary $\check{\partial} V$}

As we commented before, the corresponding arguments and results for the past c-completion are deduced in a totally analogous way. Summarizing Theorems 5.5 and 5.16 we have:

Theorem 5.18 Let $V=(\mathbb{R} \times M, g)$ be a spacetime as in (4.1) such that $g_{c l} \prec_{0}$ $g \prec_{0} g_{o p}$ holds. If the integral condition

$$
\int_{-\infty}^{0}\left(\frac{1}{\alpha(s)}-1\right) d s<\infty
$$

holds, then the map

$$
\begin{array}{cccc}
\check{j}: & \check{V}_{c l} & \rightarrow & \check{V} \\
F_{c l} & \mapsto & I^{+}\left(F_{c l}\right)
\end{array}
$$


is injective. So, $\check{V}$ contains a cone of base $M_{B}^{-}$and apex $i^{-}$.

Moreover, if additionally $M_{C}^{-}$is locally compact and $d_{Q}^{-}$is a generalized distance, then the map

$$
\check{\mathcal{J}}=\check{\Pi} \circ \check{j}: \check{V}_{c l} \rightarrow \check{V} / \sim_{s t}
$$

is bijective and continuous. When $\check{V} / \sim_{s t}$ is also Hausdorff then $\check{\mathcal{J}}$ is an homeomorphism.

Remark 5.19 According to Corollary 5.6, we can establish the following assertions under the general hypotheses of Theorem 5.18. (1) if $\left(M, d^{-}\right)$is bounded then $\check{V} V$ contains a cone of base $\partial_{C}^{-} M$ and apex $i^{-}$; if, in addition, $\left(M, d^{-}\right)$is complete (thus, compact), the past c-boundary is $i^{-} ;(2)$ if $\left(M, d^{-}\right)$is complete and 5.15 holds, the past c-boundary contains a cone of base $\partial_{\mathcal{B}}^{-} M$ and apex $i^{-}$(recall Remark 4.8).

\section{The (total) c-boundary $\partial V$}

Now, we are ready to study the total c-boundary of $V=(\mathbb{R} \times M, g)$.

\section{1 $\partial V$ as a point set}

As in the study of partial boundaries (recall Theorems 5.5 and 5.18), firstly our aim is to introduce and analyze a map of the form

$$
\bar{j}: \bar{V}_{c l} \longrightarrow \bar{V} .
$$

In a first attempt, one could try to define $\bar{j}\left(\left(P_{c l}, F_{c l}\right)\right)=\left(\hat{j}\left(P_{c l}\right), \check{j}\left(F_{c l}\right)\right)$. However, it is not true in general that $P_{c l} \sim_{S} F_{c l}$ implies $\hat{j}\left(P_{c l}\right)\left(=I^{-}\left(P_{c l}\right)\right) \sim_{S} \breve{j}\left(F_{c l}\right)(=$ $\left.I^{+}\left(F_{c l}\right)\right)$, and so, we cannot ensure that $\left(P_{c l}, F_{c l}\right) \in \bar{V}_{c l}$ implies $\left(\hat{j}\left(P_{c l}\right), \breve{j}\left(F_{c l}\right)\right) \in \bar{V}$. The reason of this problem is that, even if the choices for $\hat{j}$ and $\breve{j}$ are canonical, there exists potential alternatives (see Remark 5.8 ), and the ambiguity derived from these alternatives yields pathological properties when the total c-completion is studied. Therefore, in order to deal with $\bar{j}$, we have to look into the different alternatives for $\hat{j}$ and $\breve{j}$. With this aim, let us consider the following two lemmas.

Lemma 6.1 Let $\left(P_{c l}, F_{c l}\right) \in \bar{V}_{c l}$ be such that $P_{c l}$ and $F_{c l}$ are associated to the same $p=\left(\Omega, x^{s}\right) \in \mathbb{R} \times M_{C}^{s}$, i.e. $P_{c l}=P_{c l}\left(d_{p}^{+}\right)$and $F_{c l}=F_{c l}\left(d_{p}^{-}\right)$. Then, there exist $P \in \hat{V}$ and $F \in \check{V}$ with $P \neq \emptyset \neq F$ such that $P \sim_{S} F, P \in \hat{\Pi}^{-1}\left(\hat{\mathcal{J}}\left(P_{c l}\right)\right)$ and $F \in$ $\check{\Pi}^{-1}\left(\check{\mathcal{J}}\left(F_{c l}\right)\right)$. In the case that $x^{s} \in M$, then $P=I^{-}\left(\left(\Omega, x^{s}\right)\right)$ and $F=I^{+}\left(\left(\Omega, x^{s}\right)\right)$.

Proof. The last assertion is trivial, so we can focus directly on the case $x^{s} \in \partial_{C}^{s} M$, and thus, $\left(P_{c l}, F_{c l}\right) \in \partial_{c l} V$. As $P_{c l} \sim_{S} F_{c l}$ and $g_{c l} \prec g_{o p}$, the following inclusions hold:

$$
P_{c l} \subset \downarrow_{c l} F_{c l} \subset \downarrow_{o p} F_{c l}, \quad F_{c l} \subset \uparrow_{c l} P_{c l} \subset \uparrow_{o p} P_{c l} .
$$

Taking into account that $g_{c l} \prec_{0} g$, we also have:

$$
I^{-}\left(P_{c l}\right) \subset \downarrow I^{+}\left(F_{c l}\right) \quad I^{+}\left(F_{c l}\right) \subset \uparrow I^{-}\left(P_{c l}\right) .
$$


Therefore, since $I^{-}\left(P_{c l}\right)$ and $I^{+}\left(F_{c l}\right)$ are an IP and an IF, resp., for $g$, there exist some TIP $P$ and some TIF $F$ such that

$$
I^{-}\left(P_{c l}\right) \subset P, \quad I^{+}\left(F_{c l}\right) \subset F \quad \text { and } \quad P \sim_{S} F
$$

(for instance, take $P$ being maximal IP in $\downarrow I^{+}\left(F_{c l}\right)$ containing $I^{-}\left(P_{c l}\right)$ and $F$ being maximal IF in $\uparrow P$ containing $\left.I^{+}\left(F_{c l}\right)\right)$. Next, we are going to prove that $P \in \hat{\Pi}^{-1}\left(\left(\hat{\mathcal{J}}\left(P_{c l}\right)\right)\right.$ and $F \in \check{\Pi}^{-1}\left(\breve{\mathcal{J}}\left(F_{c l}\right)\right)$. Taking into account that $g \prec_{0} g_{o p}, 6.1$ implies:

$$
\begin{aligned}
& I_{o p}^{-}\left(P_{c l}\right) \subset I_{o p}^{-}(P) \subset \downarrow I_{o p}^{+}(F) \subset \downarrow I_{o p}^{+}\left(F_{c l}\right) \\
& I_{o p}^{+}\left(F_{c l}\right) \subset I_{o p}^{+}(F) \subset \uparrow I_{o p}^{-}(P) \subset \uparrow I_{o p}^{-}\left(P_{c l}\right) .
\end{aligned}
$$

In fact, for the first inclusion of the first line note that $P_{c l} \subset P$ implies $I_{o p}^{-}\left(P_{c l}\right) \subset$ $I_{o p}^{-}(P)$. For the second inclusion, $P \sim_{S} F$ implies $P \subset \downarrow F$, and thus, $I_{o p}^{-}(P) \subset \downarrow_{o p}$ $I_{o p}^{+}(F)$. Finally, for the third inclusion, $F_{c l} \subset F$ implies $I_{o p}^{+}\left(F_{c l}\right) \subset I_{o p}^{+}(F)$, and so, $\uparrow_{o p} I_{o p}^{+}(F) \subset \uparrow_{o p} I_{o p}^{+}\left(F_{c l}\right)$. The inclusions in the second line are deduced analogously.

From the construction, $I_{o p}^{-}\left(P_{c l}\right)$ and $I_{o p}^{+}\left(F_{c l}\right)$ are associated to the same pair $\left(\Omega, x^{s}\right)$, and so, $I_{o p}^{-}\left(P_{c l}\right) \sim_{S} I_{o p}^{+}\left(F_{c l}\right)$. From previous inclusions, $I_{o p}^{-}(P)=I_{o p}^{-}\left(P_{c l}\right)$ and $I_{o p}^{+}(F)=I_{o p}^{+}\left(F_{c l}\right)$, since, otherwise, the maximality of $I_{o p}^{-}\left(P_{c l}\right)$ and $I_{o p}^{+}\left(F_{c l}\right)$ would be violated (recall (2.1)). This joined to the fact that $P_{c l} \subset P$ and $F_{c l} \subset F$ (recall 6.1) gives $P \in \hat{\Pi}^{-1}\left(\left(\hat{\mathcal{J}}\left(P_{c l}\right)\right)\right.$ and $F \in \check{\Pi}^{-1}\left(\check{\mathcal{J}}\left(F_{c l}\right)\right)$, as required.

Lemma 6.2 Suppose that $d_{Q}^{+}$is a generalized distance. If $\left(P_{c l}, F_{c l}\right) \in \partial_{c l} V$ satisfies $F_{c l}=\emptyset\left(\right.$ resp. $\left.P_{c l}=\emptyset\right)$, then $\left(I^{-}\left(P_{c l}\right), \emptyset\right) \in \partial V\left(\right.$ resp. $\left.\left(\emptyset, I^{+}\left(F_{c l}\right)\right) \in \partial V\right)$.

Proof. Suppose $F_{c l}=\emptyset$ (thus, $P_{c l} \neq \emptyset$ ). As $d_{Q}^{+}$is a generalized distance, there exists a timelike curve $\gamma:[a, \infty) \rightarrow V, \gamma(t)=(t, c(t))$, such that $P_{c l}=I_{c l}^{-}(\gamma)=P_{c l}\left(b_{c, c l}^{+}\right)$ (recall the second point of the Point Set structure in Theorem 3.4). In particular, from $g \prec g_{o p}$,

$$
\uparrow I^{-}\left(P_{c l}\right) \subset \uparrow_{o p} I_{o p}^{-}\left(P_{c l}\right)=\emptyset .
$$

Therefore, $\left(I^{-}\left(P_{c l}\right), \emptyset\right) \in \partial V$.

Observe that, under the hypothesis of $d_{Q}^{+}$being a generalized distance, previous lemmas include all the possibilities for pairs in $\partial_{c l} V$ (recall the second point of the Point Set structure in Theorem 3.4. So, we are able to establish the following result:

Proposition 6.3 Let $V$ be a spacetime as in (4.1) such that $g_{c l} \prec_{0} g \prec_{0} g_{o p}$, and assume that (5.3), 5.15) hold and $d_{Q}^{+}$is a generalized distance. Consider any map $\bar{j}: \bar{V}_{c l} \rightarrow \bar{V}$ given by:

$$
\bar{j}\left(\left(P_{c l}, F_{c l}\right)\right):= \begin{cases}\left(I^{-}\left(P_{c l}\right), \emptyset\right) & \text { if } F_{c l}=\emptyset \\ \left(\emptyset, I^{+}\left(F_{c l}\right)\right) & \text { if } P_{c l}=\emptyset \\ \left(P_{0}, F_{0}\right) & \text { otherwise }\end{cases}
$$

where $\left(P_{0}, F_{0}\right)$ is any particular choice of pair obtained from Lemma 6.1 (so that, $P_{0} \in \hat{\Pi}^{-1}\left(\hat{\mathcal{J}}\left(P_{c l}\right)\right)$ and $\left.F_{0} \in \check{\Pi}^{-1}\left(\check{\mathcal{J}}\left(P_{c l}\right)\right)\right)$. Then, $\bar{j}$ is well defined and injective. 
Proof. Observe that, if $d_{Q}^{+}$is a generalized distance, any $\left(P_{c l}, F_{c l}\right) \in \bar{V}_{c l}$ should fall under the conditions of Lemma 6.1 or Lemma 6.2 (recall Theorem 3.4, the second point about the Point Set structure $)$, and so, $\bar{j}\left(\left(P_{c l}, F_{c l}\right)\right) \in \bar{V}$, i.e., $\bar{j}$ is well defined.

For the injectivity, assume that $\bar{j}\left(\left(P_{c l}, F_{c l}\right)\right)=(P, F)=\bar{j}\left(\left(P_{c l}^{\prime}, F_{c l}^{\prime}\right)\right)$. If $P \neq \emptyset$ (the case $F \neq \emptyset$ is analogous), we deduce from the construction that

$$
P_{o p}=I_{o p}^{-}\left(P_{c l}\right)=I_{o p}^{-}(P)=I_{o p}^{-}\left(P_{c l}^{\prime}\right)=P_{o p}^{\prime}
$$

So, applying Proposition 5.3 (2) we have that $P_{c l}=P_{c l}^{\prime}$. Finally, as $d_{Q}^{+}$is a generalized distance, Theorem 3.4 ensures that $F_{c l}=F_{c l}^{\prime}$.

Recall that in the case of partial boundaries we made a canonical choice for $\hat{j}$ and $\breve{j}$, but alternative choices may deserve to be taken into account (recall Remark 5.8). Here, $\bar{j}$ is explicitly constructed by using any of the possible $\left(P_{0}, F_{0}\right)$ provided by Lemma 6.1. In order to avoid this ambiguity, as well as possible problems with the continuity of $\bar{j}$, we will extend the concept of strains to the c-completion (recall Definition 5.11). In this sense, let us associate first, to each $\left(P_{c l}, F_{c l}\right) \in \bar{V}_{c l}$, the subset $S T\left(\left(P_{c l}, F_{c l}\right)\right) \subset \bar{V}$ defined as follows:

$$
(P, F) \in S T\left(\left(P_{c l}, F_{c l}\right)\right) \Longleftrightarrow\left\{\begin{array}{lll}
\text { if } P \neq \emptyset \neq P_{c l} & \text { then } & P \in \hat{\Pi}^{-1}\left(\hat{\mathcal{J}}\left(P_{c l}\right)\right) \\
\text { if } F \neq \emptyset \neq F_{c l} & \text { then } & F \in \check{\Pi}^{-1}\left(\tilde{\mathcal{J}}\left(F_{c l}\right)\right) \\
\text { if } P_{c l}=\emptyset & \text { then } & P=\emptyset \\
\text { if } F_{c l}=\emptyset & \text { then } & F=\emptyset
\end{array}\right.
$$

In particular, if namely $(\emptyset, F) \in S T\left(\left(\emptyset, F_{c l}\right)\right)$, then $F \neq \emptyset \neq F_{c l}$, and so, $F \in$ $\check{\Pi}^{-1}\left(\check{\mathcal{J}}\left(F_{c l}\right)\right)$.

Lemma 6.4 If $\left(P_{c l}, F_{c l}\right) \neq\left(P_{c l}^{\prime}, F_{c l}^{\prime}\right)$ then $S T\left(\left(P_{c l}, F_{c l}\right)\right) \cap S T\left(\left(P_{c l}^{\prime}, F_{c l}^{\prime}\right)\right)=\emptyset$.

Proof. Assume the existence of some

$$
(P, F) \in S T\left(\left(P_{c l}, F_{c l}\right)\right) \cap S T\left(\left(P_{c l}^{\prime}, F_{c l}^{\prime}\right)\right) \neq \emptyset, \quad \text { with } P \neq \emptyset,
$$

(the case $F \neq \emptyset$ is analogous). Then, $P \in \hat{\Pi}^{-1}\left(\hat{\mathcal{J}}\left(P_{c l}\right)\right) \cap \hat{\Pi}^{-1}\left(\hat{\mathcal{J}}\left(P_{c l}^{\prime}\right)\right)$ and, by the injectivity of $\hat{\Pi}$ and $\hat{\mathcal{J}}$, necessarily $P_{c l}=P_{c l}^{\prime}$. On the other hand, as $d_{Q}^{+}$is a generalized distance, the c-completion $\bar{V}_{c l}$ is simple as a point set (recall Definition 2.3. Therefore, from $P_{c l}=P_{c l}^{\prime}$, we deduce that $\left(P_{c l}, F_{c l}\right)=\left(P_{c l}^{\prime}, F_{c l}^{\prime}\right)$, as desired.

Now we are in conditions to introduce the following definition for strains in $\bar{V}$ by means of a relation of equivalence (observe that reflexivity and symmetry are straightforward, while transitivity is a consequence of previous lemma).

Definition 6.5 Let $\sim_{s t}$ be the relation of equivalence on $\bar{V}$ defined by:

$(P, F) \sim_{s t}\left(P^{\prime}, F^{\prime}\right) \Longleftrightarrow\left\{\begin{array}{l}(P, F)=\left(P^{\prime}, F^{\prime}\right), \text { or } \\ (P, F),\left(P^{\prime}, F^{\prime}\right) \in S T\left(\left(P_{c l}, F_{c l}\right)\right) \quad \text { for some }\left(P_{c l}, F_{c l}\right) \in \bar{V}_{c l} .\end{array}\right.$

The (non-trivial) classes of equivalence in $\bar{V} / \sim_{s t}$ will be called (total) strains. We will denote by $\Pi: \bar{V} \rightarrow \bar{V} / \sim_{\text {st }}$ the natural projection onto the quotient. 

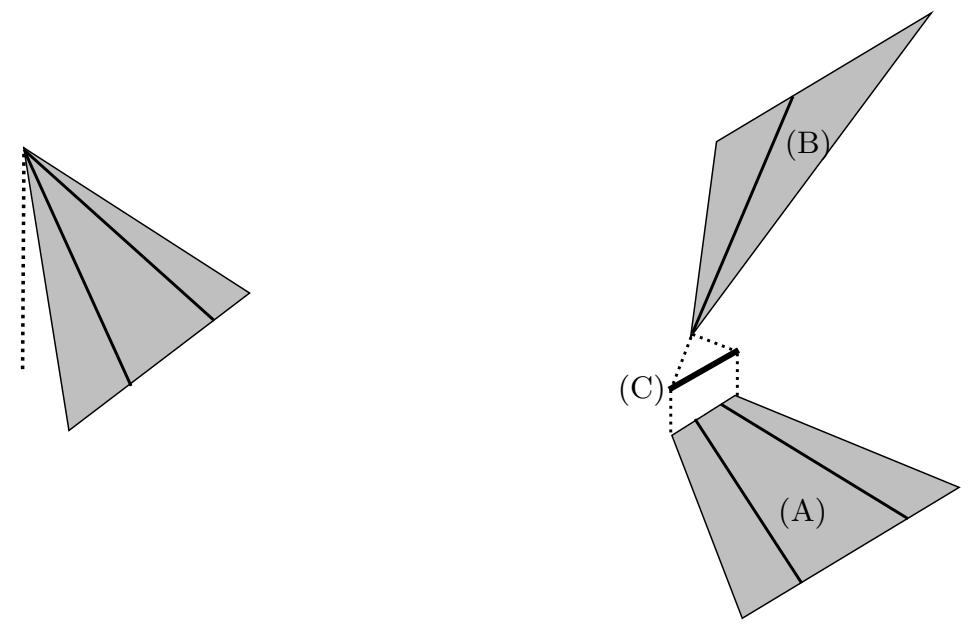

Figure 5: Two examples of (total) strains.

The left figure shows a tame possibility for the strain. It is formed by a foliation of lightlike curves ending at the same point. All the boundary points in this strain are of the form $(P, \emptyset)$.

The right figure shows a wilder possibility. The strain is formed by three connected components: Two of them, labeled by (A) and (B), are foliated by lightlike curves, and the lightlike curves in the first one do not necessarily end at the same point. The third one is the segment $(\mathrm{C})$. The boundary points in $(\mathrm{A})$ and $(\mathrm{B})$ are of the form $(P, \emptyset)$ and $(\emptyset, F)$ resp., while the points in the segment $(\mathrm{C})$ are of the form $(P, F)$ with $P \neq \emptyset \neq F$. 
As in the case of the partial boundaries, the following result assures that the total boundary contains $\partial_{c l} V$ as a point set. Its proof is straightforward from Lemma 6.4 .

Theorem 6.6 Let $V=(\mathbb{R} \times M, g)$ be a spacetime as in (4.1) such that $g_{c l} \prec_{0} g \prec_{0}$ $g_{\text {op }}$. If $d_{Q}^{+}$is a generalized distance and (5.3), 5.15) hold, then the map

$$
\mathcal{J}: \bar{V}_{c l} \rightarrow \bar{V} / \sim_{s t}, \quad\left(P_{c l}, F_{c l}\right) \mapsto \Pi\left(\bar{j}\left(\left(P_{c l}, F_{c l}\right)\right)\right)
$$

is injective.

Taking into account the structure of the c-boundary in terms of lines (recall Theorem 3.4 , we can also define quotient lines in $\partial V / \sim_{s t}$ of the following type:

$$
\mathcal{J}\left(\mathcal{L}\left(P_{c l}, F_{c l}\right)\right):=\left\{\mathcal{J}\left(\left(P_{c l}\left(b_{c, c l}^{+}+k\right), F_{c l}\left(b_{c^{\prime}, c l}^{-}+k\right)\right)\right), k \in \mathbb{R}\right\},
$$

where $\left(P_{c l}, F_{c l}\right) \in \partial_{c l} V$ with $P_{c l} \equiv P_{c l}\left(b_{c, c l}^{+}\right), F_{c l}=F_{c l}\left(b_{c^{\prime}, c l}^{-}\right)$.

Such a definition suggests a natural lifting to $\partial V$ defined by $\bar{j}$ in the following way

$$
\bar{j}\left(\mathcal{L}\left(P_{c l}, F_{c l}\right)\right):=\left\{\bar{j}\left(P_{c l}\left(b_{c, c l}^{+}+k\right), F_{c l}\left(b_{c^{\prime}, c l}^{-}+k\right)\right), k \in \mathbb{R}\right\} .
$$

This lifting preserves properties of the original line $\mathcal{L}\left(P_{c l}, F_{c l}\right)$ with independence of the choice of $\bar{j}$ in 6.2. For example, as we will see in the next section, it preserves the causal structure. Let us introduce some notation for these elements:

Definition 6.7 Consider a line $\mathcal{L}\left(P_{c l}, F_{c l}\right) \subset \partial_{c l} V$ for some $\left(P_{c l}, F_{c l}\right) \in \partial_{c l} V$ and consider the quotient line $\mathcal{J}\left(\mathcal{L}\left(P_{c l}, F_{c l}\right)\right) \subset \partial V / \sim_{s t}$ (see 6.5)). The set $\bar{j}\left(\mathcal{L}\left(P_{c l}, F_{c l}\right)\right)$, defined in 6.6), will be called the column of the quotient line. For each $x \in \mathcal{J}\left(\mathcal{L}\left(P_{c l}, F_{c l}\right)\right), \Pi^{-1}(x)$ will be a bone of the quotient line. Finally, we will call the set $\Pi^{-1}\left(\mathcal{J}\left(\mathcal{L}\left(P_{c l}, F_{c l}\right)\right)\right)$ skeleton (see Figure 3).

Finally, recalling the point set structure of $\partial_{c l} V$ described in Theorem 3.4 .

Corollary 6.8 Under the hypotheses of Theorem 6.6, the boundary $\partial V$ contains a skeleton (and thus, at least, a column) for each line of $\partial_{c l} V$.

\subsection{Causality}

For the causal structure, note that we have not already defined a causal relation in the space $\bar{V} / \sim_{s t}$. In fact, it is not expected in general to obtain a complete satisfactory definition of causal relation in this kind of spaces. The reason is simple: we have not ensured that the relation between representatives of different strains is independent of the choice of such a representatives. However, for $\left(P_{c l}, F_{c l}\right) \in \bar{V}$, the line $\mathcal{J}\left(\mathcal{L}\left(P_{c l}, F_{c l}\right)\right)$ has a lifting, the column, which satisfies the following property.

Theorem 6.9 Let $V=(\mathbb{R} \times M, g)$ be a spacetime as in (4.1) with $g_{c l} \prec_{0} g \prec_{0}$ $g_{o p}$. Assume that $d_{Q}^{+}$is a generalized distance and 5.3), (5.15) hold. For every $\left(P_{c l}, F_{c l}\right) \in \partial_{c l} V$, the column

$$
\bar{j}\left(\mathcal{L}\left(P_{c l}, F_{c l}\right)\right) \text { is } \begin{cases}\text { horismotic } & \text { if, either } P_{c l}=\emptyset, \text { or } F_{c l}=\emptyset \\ \text { timelike } & \text { otherwise. }\end{cases}
$$


Proof. Assume that $P_{c l}=P_{c l}\left(b_{c, c l}^{+}\right)$and $F_{c l}=F_{c l}\left(b_{c^{\prime}, c l}^{-}\right)$. Given two pairs $\left(P_{c l}^{i}, F_{c l}^{i}\right) \in$ $\mathcal{L}\left(P_{c l}, F_{c l}\right), i=1,2$, necessarily $P_{c l}^{i} \equiv P_{c l}\left(b_{c}^{+}+k_{i}\right), F_{c l}^{i} \equiv F_{c l}\left(b_{c^{\prime}}^{-}+k_{i}\right)$ with, say, $k_{1}<k_{2}$. Then, one has two possibilities:

- Case $F_{c l}=\emptyset \neq P_{c l}$ (the case $F_{c l} \neq \emptyset=P_{c l}$ is analogous). From Theorem 3.4 (with $\tilde{\alpha} \equiv 1), P_{c l}^{1} \subset P_{c l}^{2}$. Hence, $I^{-}\left(P_{c l}^{1}\right) \subset I^{-}\left(P_{c l}^{2}\right)$, and thus $\left(I^{-}\left(P_{c l}^{1}\right), \emptyset\right)$ is causally, but not timelike, related to $\left(I^{-}\left(P_{c l}^{2}\right), \emptyset\right)$. In conclusion, the set $\bar{j}\left(\mathcal{L}\left(P_{c l}, F_{c l}\right)\right)$ is horismotic.

- Case $P_{c l} \neq \emptyset \neq F_{c l}$. As $\mathcal{L}\left(P_{c l}, F_{c l}\right)$ is timelike (see Theorem 3.4 , $P_{c l}^{2} \cap F_{c l}^{1} \neq$ $\emptyset$. Consider $\left(P^{i}, F^{i}\right)=\bar{j}\left(P_{c l}^{i}, F_{c l}^{i}\right)$, with $i=1,2$. From the definition of $\bar{j}$, $P_{c l}^{i} \subset P^{i}, F_{c l}^{i} \subset F^{i}$ (recall Lemma 6.1). Hence, $\emptyset \neq F_{c l}^{1} \cap P_{c l}^{2} \subset F^{1} \cap P^{2}$, and thus, $\bar{j}\left(\mathcal{L}\left(P_{c l}, F_{c l}\right)\right)$ is timelike.

Even though there is not a unique choice for $\bar{j}$, Theorem 6.9 is independent of this choice. So, the following definition grasps the causal structure of the quotient $\bar{V} / \sim_{s t}$.

Definition 6.10 The quotient line $\mathcal{J}\left(\mathcal{L}\left(P_{c l}, F_{c l}\right)\right)$ is: horismotic if either $P_{c l}=\emptyset$, or $F_{c l}=\emptyset$; and timelike otherwise.

\subsection{Topology}

Now, we are ready to establish and prove our main result.

Theorem 6.11 Let $V=(\mathbb{R} \times M, g)$ be a spacetime as in $(4.1)$ with $g_{c l} \prec_{0} g \prec_{0} g_{o p}$. Assume that $d_{Q}^{+}$is a generalized distance, $M_{C}^{s}$ is locally compact and (5.3), (5.15) hold. Then, the map $\mathcal{J}: \bar{V}_{c l} \rightarrow \bar{V} / \sim_{\text {st }}$ in (6.4) is injective and continuous.

If, in addition, $\bar{V} / \sim_{s t}$ is Hausdorff, then $\mathcal{J}$ is an homeomorphism.

Proof of the first statement. The injectivity of $\mathcal{J}$ was proved in Theorem 6.6. For the continuity of $\mathcal{J}$, assume that $\left(P_{c l}, F_{c l}\right) \in L\left(\left\{\left(P_{c l}^{n}, F_{c l}^{n}\right)\right\}\right)$ and let us prove that $\left\{\mathcal{J}\left(\left(P_{c l}^{n}, F_{c l}^{n}\right)\right)\right\}_{n}$ converges to $\mathcal{J}\left(\left(P_{c l}, F_{c l}\right)\right)$. By contradiction, suppose that there exists some neighborhood $U$ of $\mathcal{J}\left(\left(P_{c l}, F_{c l}\right)\right)$ such that, up to a subsequence, $\mathcal{J}\left(\left(P_{c l}^{n}, F_{c l}^{n}\right)\right) \notin U$ for any $n$. Then, the open set $\Pi^{-1}(U)$, which contains all the elements in $\Pi^{-1}\left(\mathcal{J}\left(\left(P_{c l}, F_{c l}\right)\right)\right)$, does not contain any element in $\Pi^{-1}\left(\mathcal{J}\left(\left(P_{c l}^{n}, F_{c l}^{n}\right)\right)\right)$ for any $n$. Now, suppose that $P_{c l} \neq \emptyset$ (the case $F_{c l} \neq \emptyset$ is analogous), and thus, $P_{c l} \in \hat{L}\left(\left\{P_{c l}^{n}\right\}\right)$. Note that $P_{c l}^{n} \neq \emptyset$ for $n$ big enough. From Lemma 5.14, any sequence $\left\{P^{n}\right\}$, with $P^{n} \in \hat{\Pi}^{-1}\left(\hat{\mathcal{J}}\left(P_{c l}^{n}\right)\right)$ for all $n$, admits some subsequence $\left\{P^{n_{k}}\right\}_{k}$ converging to some $P \in \hat{\Pi}^{-1}\left(\hat{\mathcal{J}}\left(P_{c l}\right)\right)$. Consider the following two possibilities:

- Case $F_{c l}=\emptyset$. Then, according to Lemma 6.2. $(P, \emptyset) \in \Pi^{-1}\left(\mathcal{J}\left(\left(P_{c l}, \emptyset\right)\right)\right)$, and so, $\left\{\left(P^{n_{k}}, F^{n_{k}}\right)\right\}_{k} \rightarrow(P, \emptyset)$ with the chronological topology, where $F^{n_{k}}$ is chosen such that $\left(P^{n_{k}}, F^{n_{k}}\right) \in \partial V$ for every $k$.

- Case $F_{c l} \neq \emptyset$. Then, $P_{c l}^{n} \neq \emptyset \neq F_{c l}^{n}$ for $n$ big enough. From Lemma 6.1. one can take $\left(P^{n}, F^{n}\right) \in \Pi^{-1}\left(\mathcal{J}\left(\left(P_{c l}^{n}, F_{c l}^{n}\right)\right)\right)$ with $P^{n} \neq \emptyset \neq F^{n}$. From Lemma 5.14 (and its dual version for the past), there exists some subsequence $\left\{\left(P^{n_{k}}, F^{n_{k}}\right)\right\}_{k}$ converging to some $(P, F) \in \Pi^{-1}\left(\mathcal{J}\left(\left(P_{c l}, F_{c l}\right)\right)\right)$. 
In both cases one deduces the existence of some subsequence $\left\{\left(P^{n_{k}}, F^{n_{k}}\right)\right\}_{k}$, with $\left(P^{n_{k}}, F^{n_{k}}\right) \in \Pi^{-1}\left(\mathcal{J}\left(\left(P_{c l}^{n_{k}}, F_{c l}^{n_{k}}\right)\right)\right)$, converging to some $(P, F) \in \Pi^{-1}\left(\mathcal{J}\left(\left(P_{c l}, F_{c l}\right)\right)\right)$. So, $\left(P^{n_{k}}, F^{n_{k}}\right) \in \Pi^{-1}(U)$ for $k$ big enough, a contradiction.

In general, the result claimed in this first statement of previous theorem is optimal. In fact, the structure of the c-completion of the spacetime $(V, g)$ could be very complicated, and it may differ radically from the c-completion of the stationary one (even after the identification of the strains). So, in order to go further, it is necessary to impose additional conditions which simplify this structure. In this sense, the Hausdorff character of the quotient space $\bar{V} / \sim_{s t}$ imposed in the last statement of Theorem 6.11 becomes natural (see [3, 6, 7] for other works where the Hausdorff character of the c-completion has been analyzed).

In order to prove the last statement of Theorem 6.11, first we establish the following technical lemma:

Lemma 6.12 Assume that $d_{Q}^{+}$is a generalized distance, $M_{C}^{s}$ is locally compact and $\bar{V} / \sim_{s t}$ is Hausdorff. If $(P, F) \in \partial V$ with $P \neq \emptyset \neq F$, then $P_{o p}\left(=I_{o p}^{-}(P)\right) \sim_{S}$ $F_{o p}\left(=I_{o p}^{+}(F)\right)$.

Proof. Let $\gamma_{P}:[a, \Omega) \rightarrow V, \gamma_{P}(t)=\left(t, c_{P}(t)\right)$ and $\gamma_{F}:[\bar{a},-\bar{\Omega}) \rightarrow V, \gamma_{F}(t)=$ $\left(-t, c_{F}(t)\right)$ timelike for $g$ such that $P=I^{-}\left(\gamma_{P}\right)$ and $F=I^{+}\left(\gamma_{F}\right)$. From $\uparrow P \neq$ $\emptyset \neq \downarrow F$ (recall that $\emptyset \neq P \sim_{S} F \neq \emptyset$ ), it is $\Omega,-\bar{\Omega}<\infty$. Hence, $c_{P}, c_{F}$ must converge to some $x_{0}, x_{1} \in M_{C}^{s}$, respectively (recall that $\gamma_{P}, \gamma_{F}$ are timelike curves for $g_{o p}$, the discussion above (3.7), Remark 4.8 and the fact that $d_{Q}^{+}$is a generalized distance). In particular, $P_{o p}=P_{o p}\left(d_{\left(\Omega_{o p}, x_{0}\right)}^{+}\right)$and $F_{o p}=F_{o p}\left(d_{\left(\bar{\Omega}_{o p}, x_{1}\right)}^{-}\right)$, where $\Omega_{o p}$ and $\bar{\Omega}_{o p}$ are defined as in $(5.10)$. In order to show that $P_{o p} \sim_{S} F_{o p}$, we are going to prove that $\left(\Omega_{o p}, x_{0}\right)=\left(\bar{\Omega}_{o p}, x_{1}\right)$ (recall Remark 3.3 assertion (i)), or, equivalently, $\left(\Omega, x_{0}\right)=\left(\bar{\Omega}, x_{1}\right)$.

Assume by contradiction that $\Omega \neq \bar{\Omega}$, and thus, $\Omega<\bar{\Omega}$ (otherwise, $P \not \subset \downarrow F$ ). From $P \sim_{S} F$, we ensure the existence of sequences $\left\{t_{n}\right\} \subset[a, \Omega)$ and $\left\{s_{n}\right\} \subset$ $[\bar{a},-\bar{\Omega})$ with $t_{n} \nearrow \Omega, s_{n} \nearrow-\bar{\Omega}$ and $\gamma_{P}\left(t_{n}\right) \ll \gamma_{F}\left(s_{n}\right)$ for all $n \in \mathbb{N}$. Denote by $\sigma_{n}:\left[t_{n}, s_{n}\right] \rightarrow V, \sigma_{n}(s)=\left(s, c_{n}(s)\right)$ a timelike curve joining $\gamma_{P}\left(t_{n}\right)$ and $\gamma_{F}\left(s_{n}\right)$.

First, note that for any sequence $\left\{r_{n}\right\}$ with $r_{n} \in\left[t_{n}, s_{n}\right]$, it is $P \subset \operatorname{LI}\left(\left\{I^{-}\left(\sigma_{n}\left(r_{n}\right)\right)\right\}\right)$ and $F \subset \operatorname{LI}\left(\left\{I^{+}\left(\sigma_{n}\left(r_{n}\right)\right)\right\}\right)$. Since $P \sim_{S} F$, [6, Lemma 3.15] ensures that

$$
(P, F) \in L\left(\left\{\left(I^{-}\left(\sigma_{n}\left(r_{n}\right)\right), I^{+}\left(\sigma_{n}\left(r_{n}\right)\right)\right)\right\}\right) .
$$

In particular, from the definition of $L_{\sim_{s t}}($ recall 5.9$)$ ),

$$
[(P, F)] \in L_{\sim_{s t}}\left(\left\{\left[\left(I^{-}\left(\sigma_{n}\left(r_{n}\right)\right), I^{+}\left(\sigma_{n}\left(r_{n}\right)\right)\right)\right]\right\}\right) \text { for any }\left\{r_{n}\right\} \text { with } r_{n} \in\left[t_{n}, s_{n}\right] .
$$

So, our aim will be to find a second limit point that contradicts the Hausdorffness hypothesis. With this aim, we state the following claim (proved below).

Claim: There exist $x_{\infty} \in M_{C}^{s}, t_{\infty} \in(\Omega, \bar{\Omega})$ and a subsequence $\left\{c_{n_{k}}\right\} \subset\left\{c_{n}\right\}$ such that $\lim _{k} d_{Q}^{+}\left(c_{n_{k}}\left(t_{\infty}\right), x_{\infty}\right)=0$. 
In particular, since $d_{Q}^{+}$is a generalized distance, we obtain that $\left\{d_{\left(t_{\infty}, c_{n_{k}}\left(t_{\infty}\right)\right)}^{ \pm}\right\}$ converges pointwise to $d_{\left(t_{\infty}, x_{\infty}\right)}^{ \pm}$and so that $d_{\left(t_{\infty}, x_{\infty}\right)}^{+} \in \hat{L}\left(\left\{d_{\left(t_{\infty}, c_{n_{k}}\left(t_{\infty}\right)\right)}^{+}\right\}\right)$and $d_{\left(t_{\infty}, x_{\infty}\right)}^{-} \in \check{L}\left(\left\{d_{\left(t_{\infty}, c_{n_{k}}\left(t^{\infty}\right)\right)}^{-}\right\}\right)$(recall Proposition 3.2). Therefore, if we consider the terminal sets $\overline{P_{c l}}=P_{c l}\left(d_{\left(t_{\infty}, x_{\infty}\right)}^{+}\right), \overline{F_{c l}}=F_{c l}\left(d_{\left(t_{\infty}, x_{\infty}\right)}^{-}\right)$, the characterization of $L$ at the beginning of Section 3.3 gives:

$$
\left(\bar{P}_{c l}, \bar{F}_{c l}\right) \in L\left(\left\{\left(I_{c l}^{-}\left(\sigma_{n_{k}}\left(t_{\infty}\right)\right), I_{c l}^{+}\left(\sigma_{n_{k}}\left(t_{\infty}\right)\right)\right\}\right) .\right.
$$

Summarizing, if we apply the continuous function $\mathcal{J}$ to previous sequence, and taking into account (6.7), we deduce

$$
[(P, F)], \mathcal{J}\left(\left(\bar{P}_{c l}, \bar{F}_{c l}\right)\right) \in L_{\sim_{s t}}\left(\left\{\left(I^{-}\left(\sigma_{n_{k}}\left(t_{\infty}\right)\right), I^{+}\left(\sigma_{n_{k}}\left(t_{\infty}\right)\right)\right)\right\}\right) .
$$

So, in order to get the contradiction, it suffices to prove that $[(P, F)] \neq \mathcal{J}\left(\left(\overline{P_{c l}}, \overline{F_{c l}}\right)\right)$ (recall that $\bar{V} / \sim_{s t}$ is Hausdorff). Observe that, from the definition of $\sim_{s t}$ and $\mathcal{J}$, it is deduced directly that $\Pi^{-1}\left(\mathcal{J}\left(\left(\bar{P}_{c l}, \bar{F}_{c l}\right)\right)\right)=S T\left(\left(\bar{P}_{c l}, \bar{F}_{c l}\right)\right)$. Then, one only needs to prove that $(P, F) \notin S T\left(\left(\bar{P}_{c l}, \bar{F}_{c l}\right)\right)$ or, particularly, that $P \notin \hat{\Pi}^{-1}\left(\hat{\mathcal{J}}\left(\bar{P}_{c l}\right)\right)$, since $P \neq \emptyset \neq \bar{P}_{c l}$. But this follows from the fact that $\bar{P}_{c l} \not \subset P$. In fact, $\bar{P}_{c l}=$ $P_{c l}\left(d_{\left(t_{\infty}, x_{\infty}\right)}^{+}\right)$contains points $(t, x) \in V$ with $t>\Omega$ which cannot belong to $P=$ $I^{-}\left(\gamma_{P}\right)$ (recall that the temporal component of $\gamma_{P}(t)$ is smaller than $\Omega$ ).

Therefore, we have obtained that $\Omega=\bar{\Omega}$. Finally, as $P \subset \downarrow F$ and $g \prec_{0} g_{\text {op }}$, we have that $P_{o p} \subset \downarrow_{o p} F_{o p}$. So, taking into account that $P_{o p}=P_{o p}\left(d_{\left(\Omega_{o p}, x_{0}\right)}^{+}\right), F_{o p}=$ $F_{o p}\left(d_{\left(\Omega, x_{1}\right)}^{-}\right)$and 3.14 , we have that $P_{o p}\left(d_{\left(\Omega_{o p}, x_{0}\right)}^{+}\right) \subset P_{o p}\left(d_{\left(\Omega_{o p}, x_{1}\right)}^{+}\right)$, i.e.,

$$
d_{\left(\Omega_{o p}, x_{0}\right)}^{+} \leqslant d_{\left(\Omega_{o p}, x_{1}\right)}^{+} .
$$

Evaluating both expressions in $x_{1}$, we deduce that $d_{Q}^{+}\left(x_{1}, x_{0}\right)=0$ and, taking into account that $d_{Q}^{+}$is a generalized distance, necessarily $x_{0}=x_{1}$.

Proof of the Claim. Consider the auxiliary generalized distance,

$$
d_{\text {aux }}:\left(\mathbb{R} \times M_{C}^{s}\right) \times\left(\mathbb{R} \times M_{C}^{s}\right) \rightarrow \mathbb{R}
$$

with

$$
d_{\text {aux }}\left(\left(s_{1}, y_{1}\right),\left(s_{2}, y_{2}\right)\right)=d_{Q}^{+}\left(y_{1}, y_{2}\right)+\left|s_{1}-s_{2}\right| .
$$

Our aim is to apply [7, Theorem 5.15] to the family of curves $\left\{\sigma_{n}\right\}$. In order to apply that theorem, we have to ensure the following conditions: (i) the family of curves is defined in the same interval $I=[a, b]$ with $a<b$, (ii) $\sigma_{n}$ is an oriented equicontinuous family of functions (see [7. Definition 5.10]) and (iii) $\sigma_{n}(a)$ has an accumulation point admitting a compact neighborhood. For (i), observe that we can consider $a=\Omega$ and $b=\bar{\Omega}$, as $\Omega<\bar{\Omega}, t_{n} \nearrow \Omega$ and $s_{n} \searrow \bar{\Omega}$. For (ii), recall that the curves $\sigma_{n}(s)=\left(s, c_{n}(s)\right)$ are timelike for $g$, and then, for $g_{o p}$ (recall that $\left.g \prec_{0} g_{o p}\right)$. From 4.5 :

$$
\alpha(s) F^{+}\left(\dot{c}_{n}(s)\right)<1
$$


and so,

$$
d^{+}\left(c_{n}\left(s_{1}\right), c_{n}\left(s_{2}\right)\right) \leqslant \int_{s_{1}}^{s_{2}} F^{+}\left(\dot{c}_{n}(s)\right) d s<\int_{s_{1}}^{s_{2}} \frac{1}{\alpha(s)} d s \leqslant \frac{1}{\left.\min \right|_{\left[t_{0}, s_{0}\right]}(\alpha(s))}\left(s_{2}-s_{1}\right) .
$$

Therefore, for $s_{1}<s_{2}$ we obtain that:

$$
\begin{aligned}
d_{\text {aux }}\left(\sigma_{n}\left(s_{1}\right), \sigma_{n}\left(s_{2}\right)\right) & =\left(s_{2}-s_{1}\right)+d^{+}\left(c_{n}\left(s_{1}\right), c_{n}\left(s_{2}\right)\right) \\
& <\left(s_{2}-s_{1}\right)\left(1+\frac{1}{\left.\min \right|_{\left[t_{0}, s_{0}\right]}(\alpha(s))}\right),
\end{aligned}
$$

which imply that the family $\sigma_{n}$ is oriented equicontinuous. Finally, for (iii), recall

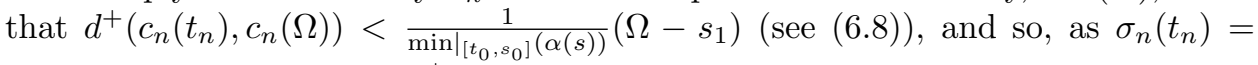
$\left(t_{n}, c_{n}\left(t_{n}\right)\right) \rightarrow\left(\Omega, x_{0}\right)$ and $d_{Q}^{+}$is a generalized distance, it is deduced that $\sigma_{n}(\Omega)=$ $\left(\Omega, c_{n}(\Omega)\right) \rightarrow\left(\Omega, x_{0}\right) \in \mathbb{R} \times M_{C}^{s}$, being $M_{C}^{s}$ locally compact.

So, from [7, Theorem 5.15] (recall that $M_{C}^{s}$ is complete) there exist a subsequence $\left\{\sigma_{n_{k}}\right\}$ and a limit curve $\sigma:[\Omega, \bar{\Omega}]$, such that $\lim _{k} d_{\text {aux }}\left(\sigma_{n_{k}}(s), \sigma(s)\right)=0$ for all $s \in[\Omega, \bar{\Omega}]$. Finally, the claim follows by taking in previous limit $s$ as $t_{\infty}:=(\Omega+\bar{\Omega}) / 2$ and denoting $\sigma\left(t_{\infty}\right)=\left(t_{\infty}, x_{\infty}\right)$.

Proof of the second statement. It remains to prove that $\mathcal{J}$ is surjective and has continuous inverse when $\bar{V} / \sim_{s t}$ is Hausdorff.

For the surjectivity of $\mathcal{J}$, consider $[(P, F)] \in \bar{V} / \sim_{s t}$ and take an element $(P, F) \in \bar{V}$ of the class $[(P, F)]$. From Lemma 5.10 (and its corresponding past version), if $P \neq \emptyset$ (resp. $F \neq \emptyset$ ) there exists a terminal set $P_{c l}$ (resp. $F_{c l}$ ) for the metric $g_{c l}$ such that $P_{c l} \subset P$ with $P_{o p}\left(:=I_{o p}^{-}(P)\right)=I_{o p}^{-}\left(P_{c l}\right)$ (resp. $F_{c l} \subset F$ with $\left.F_{o p}\left(:=I_{o p}^{+}(F)\right)=I_{o p}^{+}\left(F_{c l}\right)\right)$. If $F=\emptyset$ (the case $P=\emptyset$ is analogous), the stated properties of $P_{c l}$ ensure that $(P, \emptyset) \in S T\left(\left(P_{c l}, F_{c l}^{\prime}\right)\right)$ for any $F_{c l}^{\prime}$ such that $\bar{V}_{c l}$, and thus, $[(P, \emptyset)]=\mathcal{J}\left(\left(P_{c l}, F_{c l}^{\prime}\right)\right)$. If $P \neq \emptyset \neq F$, Lemma 6.12 ensures that $P_{o p} \sim_{S} F_{o p}$, and so, $P_{c l} \sim_{S} F_{c l}$ (both sets are associated to the same point in $\mathbb{R} \times M_{C}^{s}$, see Remark 3.3 assertion (i)). In particular, $(P, F) \in S T\left(\left(P_{c l}, F_{c l}\right)\right)$, and thus, $[(P, F)]=\mathcal{J}\left(\left(P_{c l}, F_{c l}\right)\right)$. In both cases, $[(P, F)]=\mathcal{J}\left(\left(P_{c l}, F_{c l}\right)\right)$ for some $\left(P_{c l}, F_{c l}\right) \in \bar{V}_{c l}$.

For the continuity of $\mathcal{J}^{-1}$, consider a sequence $\left\{\mathcal{J}\left(\left(P_{c l}^{n}, F_{c l}^{n}\right)\right)\right\} \subset \bar{V} / \sim_{s t}$ and some $\mathcal{J}\left(\left(P_{c l}, F_{c l}\right)\right) \in \bar{V} / \sim_{s t}$ such that:

$$
\mathcal{J}\left(\left(P_{c l}, F_{c l}\right)\right) \in L_{\sim_{s t}}\left(\mathcal{J}\left(\left(P_{c l}^{n}, F_{c l}^{n}\right)\right)\right) .
$$

By definition, there exist $\left(P^{n}, F^{n}\right) \in \mathcal{J}\left(\left(P_{c l}^{n}, F_{c l}^{n}\right)\right)$ and $(P, F) \in \mathcal{J}\left(\left(P_{c l}, F_{c l}\right)\right)$ such that:

$$
(P, F) \in L\left(\left\{\left(P^{n}, F^{n}\right)\right\}\right)
$$

We distinguish the following two cases:

- Assume that $F=\emptyset$ (the case $P=\emptyset$ is analogous). From $P \in \hat{L}\left(\left\{P^{n}\right\}\right)$ and $g \prec_{0} g_{o p}$, we deduce that $I_{o p}^{-}(P)=P_{o p} \subset \operatorname{LI}\left(\left\{P_{o p}^{n}\right\}\right)=\operatorname{LI}\left(\left\{I_{o p}^{-}\left(P^{n}\right)\right\}\right)$. In order to prove that $P_{o p} \in \hat{L}\left(\left\{P_{o p}^{n}\right\}\right)$, assume by contradiction that $P_{o p}$ is not maximal into $\operatorname{LS}\left(\left\{P_{o p}^{n}\right\}\right)$. From Lemma 5.15 there exists some $P_{o p}^{\prime}$ 
such that $P_{o p} \varsubsetneqq P_{o p}^{\prime} \in \hat{L}\left(\left\{P_{o p}^{n_{k}}\right\}\right)$ for some subsequence $\left\{P_{o p}^{n_{k}}\right\} \subset\left\{P_{o p}^{n}\right\}$. From Lemma 5.13, we obtain that $P_{c l}^{\prime} \in \hat{L}\left(\left\{P_{c l}^{n_{k}}\right\}\right)$, where $P_{c l}^{\prime}$ satisfies $I_{o p}^{-}\left(P_{c l}^{\prime}\right)=P_{o p}^{\prime}$ (recall Lemma 5.10 with $g=g_{o p}$ ). From the last assertion in Theorem 3.4 , $\left(P_{c l}^{\prime}, F_{c l}^{\prime}\right) \in L\left(\left\{\left(P_{c l}^{n_{k}}, F_{c l}^{n_{k}}\right)\right\}\right)$ for any $F_{c l}^{\prime} \in \check{V}$ such that $\left(P_{c l}^{\prime}, F_{c l}^{\prime}\right) \in \bar{V}$. The continuity of $\mathcal{J}$ ensures that

$$
\mathcal{J}\left(\left(P_{c l}^{\prime}, F_{c l}^{\prime}\right)\right) \in L_{\sim_{s t}}\left(\left\{\mathcal{J}\left(\left(P_{c l}^{n_{k}}, F_{c l}^{n_{k}}\right)\right)\right\}\right) .
$$

Therefore, from $6.9,6.10$ and the Hausdorffness of $\bar{V} / \sim_{s t}$, we have that $\mathcal{J}\left(\left(P_{c l}, F_{c l}\right)\right)=\mathcal{J}\left(\left(P_{c l}^{\prime}, F_{c l}^{\prime}\right)\right)$. So, $P_{c l}^{\prime}=P_{c l}$, and thus, $P_{o p}^{\prime}=P_{o p}$ a contradiction.

So, $P_{o p} \in \hat{L}\left(\left\{P_{o p}^{n}\right\}\right)$ and from Lemma 5.13. $P_{c l} \in \hat{L}\left(\left\{P_{c l}^{n}\right\}\right)$. Finally, from the last assertion of Theorem 3.4. $\left(P_{c l}, F_{c l}\right) \in L\left(\left\{\left(P_{c l}^{n}, F_{c l}^{n}\right)\right\}\right)$.

- Assume that $P \neq \emptyset \neq F$. From $g \prec_{0} g_{o p}$, we deduce that $P_{o p} \subset \operatorname{LI}\left(\left\{P_{o p}^{n}\right\}\right)$ and $F_{o p} \in \operatorname{LI}\left(\left\{F_{o p}^{n}\right\}\right)$. Lemma 6.12 ensures that $P_{o p} \sim_{S} F_{o p}$, and so, $\left(P_{o p}, F_{o p}\right) \in$ $L\left(\left\{\left(P_{o p}^{n}, F_{o p}^{n}\right)\right\}\right)$ (recall [6, Proposition 3.16]). Hence, from Lemma 5.13,

$$
\left(P_{c l}, F_{c l}\right) \in L\left(\left\{\left(P_{c l}^{n}, F_{c l}^{n}\right)\right\}\right) .
$$

Remark 6.13 In contraposition to the maps $\hat{\mathcal{J}}, \check{\mathcal{J}}$ for the partial boundaries, now the map $\mathcal{J}$ might be non-surjective. This is a consequence of the fact that a TIP $P$ may be S-related to multiple TIF's $F, F^{\prime}$ (and viceversa), yielding more than one point in the quotient. However, these points must be non-Hausdorff related, and so, the Hausdorffness of $\bar{V}$ (or just of $\bar{V} / \sim_{s t}$ ) would be sufficient to ensure surjectivity. At any case, if one redefine the relation of equivalence $\sim_{s t}$ by redefining previously the strains in 6.3 as follows,

$$
(P, F) \in S T^{+}\left(\left(P_{c l}, F_{c l}\right)\right) \Longleftrightarrow\left\{\begin{array}{ll}
P \in \hat{\Pi}^{-1}\left(\hat{\mathcal{J}}\left(P_{c l}\right)\right) & \text { if } P \neq \emptyset \\
F \in \check{\Pi}^{-1}\left(\check{\mathcal{J}}\left(F_{c l}\right)\right) & \text { if } P=\emptyset
\end{array},\right.
$$

the new projection $\mathcal{J}$ will be bijective (with no assumption on Hausdorffness). Note however that this definition introduces an asymmetry with respect to the temporal orientation, as it is focused on the $P$ part. In fact, we can consider a dual definition $S T^{-}$based analogously on the $F$ part, obtaining a relation of equivalence which differs from 6.11) (and (6.3)).

Finally, we remark that, independently of the approach considered, i.e. the original one for $S T$ or the time asymmetric ones $S T^{+}$and $S T^{-}$, the assertions in Remark 5.17 about the possible lack of continuity of $\mathcal{J}^{-1}$ in the non-Hausdorff case remain true.

\section{Conclusions}

By using simple standard conformally stationary spacetimes (i.e., spacetimes as in (4.1) under the hypotheses of Theorem 6.11 as model spaces, we have proved 
that isocausality yields the qualitative behavior of the c-boundary for a wide class of spacetimes. Such spacetimes include those in the general split form $V=\mathbb{R} \times M$ (see (4.1), and recall that conformally related spacetimes can be also taken into account), under the relevant hypothesis that the metric will stabilize for large values of $|t|$, in the sense of (5.3) and (5.15).

Our approach has been developed in full generality by using stationary spacetimes rather than static ones. This is remarkable, as one expects that the evolution of most isolated systems will stabilize them into stationary spacetimes. The approach is applicable as the c-boundary of standard stationary spacetimes is known [7] (including the stationary part of Kerr spacetime [4]).

Such a broad viewpoint leads to consider some technical properties of the generalized distances $d^{ \pm}$for the model spacetime and their extensions $d_{Q}^{ \pm}$to the Cauchy boundaries. But these properties will hold trivially in practical cases. For example, if we consider as model spacetime a static one $V_{c l}=\left(\mathbb{R} \times M, g_{c l}=\Lambda\left(-d t^{2}+h\right)\right)$ then no hypothesis on $d_{Q}^{ \pm}$is necessary, and we have just to check that the Cauchy boundary for $d^{+}$(a usual distance equal to $d^{-}$in the static case) is locally compact. Obviously, this is a natural property for any model spacetime - and will always hold in a realistic spacetime.

The results obtained in this paper can be extended to other cases. For example, we have assumed for the model stationary spacetime the structure $\mathbb{R} \times M$ (i.e., the timelike Killing vector field $\partial_{t}$ is complete), but one can consider also the case $I \times M$ where $I \subset \mathbb{R}$ is an interval. This is interesting, as all the causal elements are conformally invariant, and spacetimes such as Robertson-Walker ones lie in this class (up to a conformal transformation). This computation would be somewhat long, but it can be carried out with the introduced tools and, consequently, it has not been done here.

Summing up, the techniques introduced here complete the study of the c-boundary in [6, 7, by making its qualitative behavior understandable even in situations with scarce symmetries.

\section{Acknowledgements}

The authors are partially supported by the Spanish MICINN Grant MTM201018099 and Regional J. Andalucía Grant P09-FQM-4496, both with FEDER funds.

\section{References}

[1] J.K. Beem, P.E. Ehrlich, K.L. Easley, Global Lorentzian geometry, Monographs Textbooks Pure Appl. Math. 202 (Dekker Inc., New York, 1996).

[2] E. Caponio, M.A. Javaloyes, M. Sánchez, On the interplay between Lorentzian Causality and Finsler metrics of Randers type, Rev. Matem. Iberoamericana 27 (2011) 919-952. 
[3] J.L. Flores, S.G. Harris, Topology of the causal boundary for standard static spacetimes, Class. Quant. Grav. 24 (2007), no. 5, 1211-1260.

[4] J.L. Flores, J. Herrera, The c-boundary construction of spacetimes: application to stationary Kerr spacetime, VI Int. Meeting on Lorentzian Geometry, Granada, 6-9 September, 2011.

[5] J.L. Flores, J. Herrera, M. Sánchez, Isocausal spacetimes may have different causal boundaries, Class. Quant. Grav. 28175016.

[6] J.L. Flores, J. Herrera, M. Sánchez, On the final definition of the causal boundary and its relation with the conformal boundary, Adv. Theor. Math. Phys. 15 (2011) 991-1058.

[7] J.L. Flores, J. Herrera, M. Sánchez, Gromov, Cauchy and causal boundaries for Riemannian, Finslerian and Lorentzian manifolds (2010), to appear in Memoirs of the AMS. Available at arXiv:1011.1154.

[8] J.L. Flores, M. Sánchez, The causal boundary of wave-type spacetimes, J. High Energy Phys. (2008), no. 3, 036, 43 pp

[9] A. García-Parrado, M. Sánchez, Further properties of causal relationship: causal structure stability, new criteria for isocausality and counterexamples, Class. Quant. Grav. 22 (2005) 4589-4619.

[10] A. García-Parrado, J. M. M. Senovilla, Causal relationship: a new tool for the causal characterization of Lorentzian manifolds, Class. Quant. Grav. 22 (2003) 625-664.

[11] A. García-Parrado, J.M.M. Senovilla, Causal symmetries, Class. Quant. Grav. 20 (2003) L139.

[12] A. García-Parrado, J.M.M. Senovilla, General study and basic properties of causal symmetries, Class. Quant. Grav. 21 (2004) 661-696.

[13] A. García-Parrado, J. M. M. Senovilla, Causal structures and causal boundaries, Class. Quant. Grav. 22 (2005) R1-R84.

[14] R.P. Geroch, E.H. Kronheimer and R. Penrose, Ideal points in spacetime, Proc. Roy. Soc. Lond. A 237 (1972) 545-67.

[15] E. Minguzzi, M. Sánchez, The causal hierarchy of spacetimes, in Recent developments in pseudo-Riemannian Geometry (2008) 359-418. ESI Lect. in Math. Phys., European Mathematical Society Publishing House. (Available at grqc/0609119).

[16] B. O'Neill, Semi-Riemannian Geometry with applications to Relativity, Academic Press, INC, 1983.

[17] L.B. Szabados, Causal boundary for strongly causal spaces, Class. Quant. Grav. 5 (1988) 121-34. 